IPNO/TH 97-01

\title{
Relativistic effects in the pionium lifetime
}

\author{
H. Jallouli' and H. Sazdjian'] \\ Division de Physique Théoriquę, Institut de Physique Nucléaire, \\ Université Paris XI, \\ F-91406 Orsay Cedex, France
}

\begin{abstract}
The pionium decay width is evaluated in the framework of chiral perturbation theory and the relativistic bound state formalism of constraint theory. Corrections of order $O(\alpha)$ are calculated with respect to the conventional lowest-order formula, in which the strong interaction amplitude has been calculated to two-loop order with charged pion masses. Strong interaction corrections from second-order perturbation theory of the bound state wave equation are found to be of the order of $0.4 \%$. Electromagnetic radiative corrections, due to pion-photon interactions, are estimated to be of the order of $-0.1 \%$. Electromagnetic mass shift insertions in internal propagators produce a correction of the order of $0.3 \%$. The correction due to the passage from the strong interaction scattering amplitude evaluated with the mass parameter fixed at the charged pion mass to the amplitude evaluated with the mass parameter fixed at the neutral pion mass is found to be of the order of $6.4 \%$.
\end{abstract}

PACS numbers: 11.30.Rd, 12.39.Fe, 13.40.Ks, 11.10.St, 03.65.Pm.

Keywords: Chiral perturbation theory, Pion, Electromagnetic corrections, Relativistic bound state equations, Constraint theory.

\footnotetext{
${ }^{1}$ E-mail: jallouli@ipno.in2p3.fr .

${ }^{2}$ E-mail: sazdjian@ipno.in2p3.fr .

${ }^{3}$ Unité de Recherche des Universités Paris 11 et Paris 6 associée au CNRS.
} 


\section{Introduction}

The $\pi \pi$ scttering amplitude [1] represents one of the main quantities that allow confrontation of predictions of chiral perturbation theory [2, 3, 4] with experiment. Unfortunately, the lack of direct low energy data forces one to reconstruct the low energy scattering amplitude from extrapolations [5, 6, 7] from high energy data [8, 9, 10] and from indirect information coming from $K_{l 4}$ decay [1], at the price of increasing error bars on numerical values. The $20 \%$ uncertainty of the "experimental" value of the isospin zero $S$-wave scattering length, $a_{0}^{0}=0.26 \pm 0.05$ [6, 12, 13, 14], does not allow one to draw a clear-cut conclusion when the latter is compared with the theoretical prediction of standard chiral perturbation theory, which is 0.20 to the one-loop order [3] and 0.217 to the two-loop order 15].

From this viewpoint, the DIRAC experiment, which will be realized at CERN in the near future and which aims at measuring the pion scattering lengths from the lifetime of pionium $\left(\pi^{+} \pi^{-}\right.$atom) decaying into $\pi^{0} \pi^{0}$ [16, 17], might provide a decisive improvement for the above comparison. (Results on previous experiments are presented in Ref. [18.)

On the other hand, it was noticed [19] on theoretical grounds that the standard formulation of chiral perturbation theory does not provide the most general basis for an interpretation of the experimental results within the framework of QCD. It is generally assumed that the quark condensate in the chiral limit, $-<0|\bar{q} q| 0>_{0} / F_{\pi}^{2}$, has a mass scale of the order of $1 \mathrm{GeV}$, typical of massive hadron masses [3, 20]. This hypothesis, while apparently natural, has not yet received a direct experimental confirmation. In this connection, a generalized form of chiral perturbation theory was proposed [19, 21], in which the above hypothesis is relaxed and the quark condensate itself appears as an additional expansion parameter, allowing, in certain processes, its experimental evaluation. Thus, the $\pi \pi$ scattering amplitude $A(s \mid t, u)$ becomes at lowest order $O\left(p^{2}\right)$ :

$$
\begin{aligned}
A(s \mid t, u) & =\frac{1}{F_{0}^{2}}\left(s-2 \hat{m} B_{0}\right), \\
B_{0} & =-<0|\bar{q} q| 0>_{0} / F_{0}^{2}, \quad \hat{m}=\frac{m_{u}+m_{d}}{2} .
\end{aligned}
$$

$F_{0}$ is the pion decay constant $F_{\pi}$ in the chiral limit $\left(F_{\pi}=92.4 \mathrm{MeV}\right), m_{u}$ and $m_{d}$ are the masses of the quarks $u$ and $d$; Zweig rule violating effects have been neglected. The quark condensate parameter $2 \hat{m} B_{0}$ is expected to take values between 0 . and $m_{\pi}^{2}$, the latter value reproducing the standard predictions of chiral perturbation theory. 
For $2 \hat{m} B_{0} \simeq \frac{1}{2} m_{\pi}^{2}$ for instance, one finds for the scattering length $a_{0}^{0}$, including oneloop effects [22, 23], the value 0.27 , which lies $35 \%$ away from the standard value 0.20 and closer to the experimental value 0.26 . This example underlines more acutely the necessity of disposing of precise experimental informations on the pion scattering lengths to be able to distinguish between various chiral symmetry breaking schemes.

Coming back to the pionium lifetime, its lowest-order expression was established long ago in the nonrelativistic limit by various methods [24, 25, 26, 27]:

$$
\frac{1}{\tau_{0}}=\Gamma_{0}=\frac{16 \pi}{9} \sqrt{\frac{2 \Delta m_{\pi}}{m_{\pi^{+}}}} \frac{\left(a_{0}^{0}-a_{0}^{2}\right)^{2}}{m_{\pi^{+}}^{2}}\left|\psi_{+-}(0)\right|^{2}, \quad \Delta m_{\pi}=m_{\pi^{+}}-m_{\pi^{0}},
$$

where $a_{0}^{I}$ is the (dimensionless) $S$-wave scattering length in the isospin $I$ channel, usually evaluated in the literature with the charged pion mass, and $\psi_{+_{-}}(0)$ is the wave function of the pionium at the origin (in $x$-space). Characteristics of the pionium have been discussed in Ref. 28] and the relevance of its lifetime for determining chiral symmetry breaking parameters has been outlined in Ref. [29]. While the above formula provides a relationship between the pionium lifetime and the pion scattering lengths, it is desirable, for a precise theoretical interpretation of the experimental result, to have a knowledge of the possible corrections to it. This question was addressed recently in Refs. [30, 31, 32, 33].

The purpose of the present paper is to evaluate the corrections to formula (1.2) in the framework of $S U(2) \times S U(2)$ chiral perturbation theory. Apart from relativistic kinematic and mass shift corrections, they can be grouped into four categories: i) Corrections coming from second-order perturbation theory in the bound state wave equation. ii) Contributions originating from the electromagnetic radiative corrections due to pion-photon interactions. iii) Contributions coming from the electromagnetic mass shift corrections, due to quarkphoton interactions and acting through insertions of the $O\left(e^{2} p^{0}\right)$ mass shift lagrangian term in pion internal propagators. iv) Mass shift corrections with respect to the strong interaction amplitude evaluated with the charged pion mass.

The evaluation of the pionium bound state energy shift is done in the framework of constraint theory relativistic wave equations [34], which can be considered as a variant of the quasipotential approach [35, 36] and has been shown to provide a means of a covariant treatment of the QED bound state problem [37]. The above corrections to the bound state energy shift are evaluated to the relative leading order $O(\alpha)$, where $\alpha$ is the QED fine structure constant, the calculations being generally done to one loop (globally 
with respect to the strong and electromagnetic interactions).

Our results are the following. The corrections of the first type are found to be of the order of $0.4 \%$. The corrections of the second type are shown to be free of infra-red enhancement and are estimated to be of the order of $-0.1 \%$. The corrections of the third type are estimated to be of the order of $0.3 \%$. The corrections of the fourth type are found to be of the order of $6.4 \%$.

The plan of the paper is the following. In Sec. 2, the properties of the constraint theory bound state equation are briefly sketched. In Sec. 3, the latter formalism is adapted to the case of the coupled channels of the $\pi^{+} \pi^{-}$and $\pi^{0} \pi^{0}$ systems. In Sec. 4, the pionium lifetime expression in first-order of perturbation theory with respect to the strong interactions with pion mass shift is established. In Sec. 5, the corrections due to second-order perturbation theory in the bound state equation are evaluated. In Sec. 6 , the radiative corrections due to the pion-photon interaction are evaluated to one-loop order in the tree appriximation of the strong interactions. In Sec. 7, the electromagnetic mass shift corrections are calculated. A summary of the results is presented in Sec. 8 . Several details of calculations are presented in Appendices A-E.

\section{The constraint theory bound state equation}

The Bethe-Salpeter equation [38], which is the basic bound state equation in quantum field theory, has been revealed inadequate for quantitative calculations with covariant propagators. Two typical drawbacks are the following. In the nonrelativistic limit, the one-photon (or one-particle) exchange diagram yields relativistic corrections of order $1 / c$, instead of $1 / c^{2}$ [39]. In spectroscopic calculations, two-photon exchange diagrams yield spurious infra-red logarithmic singularities [40]. These effects are cancelled only with the inclusion of higher order diagrams, a feature that enormously complicates the use of the equation in perturbation theory.

In practice, the Bethe-Salpeter equation has been used in QED in the Coulomb gauge, which is a noncovariant gauge. Because of the instantaneous nature of the dominant part of the photon propagator, one is able to transform the original four-dimensional equation into a three-dimensional one and to avoid the previous difficulties 41]. However, the latter gauge has its own limitations. It neccesitates a different treatment of exchanged photons 
and of photons entering in radiative corrections. Furthermore, additional complications arise when QED is mixed with other interactions, where already covariant propagators are present.

In this respect, the wave equations obtained in the framework of constraint theory [34 have been shown to provide a satisfactory answer to the requirement of a covariant treatment of perturbation theory in the bound state problem [37.

In constraint theory the relative energy variable is eliminated by means of a constraint equation. For a two-particle system this is generally chosen in the form:

$$
C(P, p) \equiv\left(p_{1}^{2}-p_{2}^{2}\right)-\left(m_{1}^{2}-m_{2}^{2}\right) \approx 0
$$

The two-particle Green's function is projected on this hypersurface and then iterated around it. At its pole positions, the latter not being affected by the projection operation (2.1), one establishes a three-dimensional eigenvalue equation that takes the form:

$$
\widetilde{g}_{0}^{-1} \Psi=-\tilde{V} \Psi
$$

where $\widetilde{g}_{0}^{-1}$ is the wave equation operator, which will be specified below, and $\widetilde{V}$ is the potential, related to the renormalized off-mass shell scattering amplitude $T$ by a LippmannSchwinger type equation:

$$
\widetilde{V}=\widetilde{T}+\widetilde{V} \widetilde{g}_{0} \widetilde{T}, \quad \widetilde{T}=\left.\frac{i}{2 \sqrt{s}} T\right|_{C},
$$

where the index $C$ denotes the use of constraint (2.1) (on the external lines of $T$ ) and $s=$ $\left(p_{1}+p_{2}\right)^{2}$. ( $T$ is defined as the amputated four-point connected Green's function multiplied by the wave function renormalization factors of the external particles.) The amplitude $\widetilde{T}$ contains the usual Feynman diagrams, where the external particles are submitted to the constraint $C$. The second term in the right-hand side of the first of Eqs. (2.3) generates an iteration series, the diagrams of which are called "constraint diagrams", where the integrations, because of the presence of the factor $\widetilde{g}_{0}$, are three-dimensional, taking into account constraint $C$.

As long as perturbation theory is concerned, Eq. (2.2) is equivalent in content to the exact Bethe-Salpeter equation, with, however, a different arrangement of the perturbation series. The constraint theory wave function $\Psi$ is related to the Bethe-Salpeter wave function $\Phi$ by means of the projection of the latter on the constraint hypersurface (2.1), 
but the explicit form of this relationship will not be needed in the present work. Once Eq. (2.2) is solved with the exact potential $\tilde{V}$, the Bethe-Salpeter wave function $\Phi$ can be reconstructed, through the iteration procedure, in terms of the constraint theory wave function $\Psi$ and can be shown to satisfy the Bethe-Salpeter equation with the exact irreducible kernel $K$ and the same energy eigenvalue as that of Eq. (2.2) [37].

The choice of the operator $\widetilde{g}_{0}$ is not unique in principle, but in practice it is made on the basis of several natural requirements. The choice that is made below satisfies the following four properties: i) Correct nonrelativistic limit (Schrödinger equation). ii) Correct one-body limit. When one of the masses becomes infinite, one recovers the Dirac or Klein-Gordon equation in the presence of the static potential. iii) Correct hermiticity and unitarity properties. Potential $\widetilde{V}$ is an irreducible kernel, in the sense that it is free of singularities in the $s$-channel, at least in the elastic unitarity region: the constraint diagrams cancel the singularities of the reducible diagrams of $\widetilde{T}$. iv) Correct QED spectroscopy. In particular, the constraint diagram contributions remove all spurious singularities (in the bound state region) coming from $\widetilde{T}$. More generally, the leading effect of the sum of all $n$-photon exchange diagrams (in the absence of radiative corrections) is of order $O\left(\alpha^{2 n}\right)$, where $\alpha$ is the fine structure constant.

When constraint (2.1) is used, the Klein-Gordon operators of particles 1 and 2 become equal:

$$
\left.H_{0} \equiv\left(p_{1}^{2}-m_{1}^{2}\right)\right|_{C}=\left.\left(p_{2}^{2}-m_{2}^{2}\right)\right|_{C}=\frac{P^{2}}{4}-\frac{1}{2}\left(m_{1}^{2}+m_{2}^{2}\right)+\frac{\left(m_{1}^{2}-m_{2}^{2}\right)^{2}}{4 P^{2}}+p^{T 2} .
$$

We use the notations:

$$
P=p_{1}+p_{2}, \quad p=\frac{1}{2}\left(p_{1}-p_{2}\right), \quad X=\frac{1}{2}\left(x_{1}+x_{2}\right), \quad x=x_{1}-x_{2},
$$

and the decompositions of four-vectors into transverse and longitudinal vectors with respect to $P$ :

$$
\begin{aligned}
& q_{\mu}^{T}=q_{\mu}-\frac{q \cdot P}{P^{2}} P_{\mu}, \quad q_{\mu}^{L}=(q \cdot \hat{P}) \hat{P}_{\mu}, \quad \hat{P}_{\mu}=\frac{P_{\mu}}{\sqrt{P^{2}}}, \quad q_{L}=q \cdot \hat{P} \\
& P_{L}=\sqrt{P^{2}}, \quad r=\sqrt{-x^{T 2}} .
\end{aligned}
$$

For two spinless particles, $\widetilde{g}_{0}$ is chosen to be:

$$
\widetilde{g}_{0}=-\frac{1}{H_{0}+i \epsilon},
$$


up to possible finite renormalizations related to the finite parts of the individual particle propagator renormalizations; they will not show up, however, to the approximations used throughout this work. Furthermore, $\widetilde{g}_{0}$ undergoes a finite multiplicative renormalization by a constant factor $\left(1+\gamma_{1}\right)$ due to the off-mass shell treatment of the LippmannSchwinger type equation (2.3). The constant $\gamma_{1}$ appears from the requirement that the only $O(1 / r)$ terms in the QED potential come from the one-photon exchange diagram. Its presence amounts to multiplying the potential $\tilde{V}$ by $\left(1+\gamma_{1}\right)$ and continuing the use of expression (2.7) for $\widetilde{g}_{0}$, the constant $\gamma_{1}$ allowing the cancellation of a spurious $O\left(\alpha^{3}\right)$ term [37]. The use of this finite multiplicative constant, which tends to improve the perturbative expansion of the potential, should not, however, have an influence on physical quantities (in analogy with the presence of wave function renormalization constants).

Since in the present work we are interested by corrections of order $O(\alpha)$ to the pionium bound state energy, we can from the start consider the pure QED potential in its nonrelativistic limit (Coulomb potential) and use the corresponding nonrelativistic wave functions for the zeroth-order approximations. The pure QED corrections in the channel $\pi^{+} \pi^{-}-\pi^{+} \pi^{-}$, being of order $O\left(\alpha^{2}\right)$ [37, 42, will not be considered further.

The nonrelativistic Coulomb potential is here:

$$
V_{\text {Coul. }}=-2 \mu \frac{\alpha}{r}, \quad \mu=\frac{m_{1} m_{2}}{m_{1}+m_{2}}
$$

The rest of the potential in Eq. (2.3) will be treated as a perturbation. It contains the strong interaction part of the $\pi \pi$ interaction, as well as the interference part between strong and electromagnetic interactions.

In general, potential $\widetilde{V}$ being energy dependent, the scalar product of wave functions has a more complicated kernel than in the energy independent case [37]. The perturbation theory formulation in the case of energy dependent potentials can be found in Ref. 36] (valid for four- and three-dimensional equations). However, since in the present work the zeroth-order potential is the energy independent Coulomb potential (2.8), the scalar product that should be used in the perturbative calculations is the usual nonrelativistic one. Energy factors, present in higher-order potentials, should then be expanded around their zeroth-order values.

In the rest of this work we shall use, for the evaluation of the importance of various terms, the infra-red counting rules of the QED bound state system. Let, for a given 
process $1+2 \rightarrow 3+4, s, t, u$ be the Mandelstam variables: $s=\left(p_{1}+p_{2}\right)^{2}, t=\left(p_{1}-p_{3}\right)^{2}$, $u=\left(p_{1}-p_{4}\right)^{2}$. We also define the (c.m.) momentum operators:

$$
\begin{aligned}
b_{a b}^{2}(s) & =\frac{s}{4}-\frac{1}{2}\left(p_{a}^{2}+p_{b}^{2}\right)+\frac{\left(p_{a}^{2}-p_{b}^{2}\right)^{2}}{4 s}=-p^{T 2} \quad(a, b=1,2 \text { or } 3,4), \\
b_{0, a b}^{2}(s) & =\frac{s}{4}-\frac{1}{2}\left(m_{\pi^{a}}^{2}+m_{\pi^{b}}^{2}\right)+\frac{\left(m_{\pi^{a}}^{2}-m_{\pi^{b}}^{2}\right)^{2}}{4 s}=p_{a L}^{2}-m_{\pi^{a}}^{2}=p_{b L}^{2}-m_{\pi^{b}}^{2} .
\end{aligned}
$$

In the pionium state $\left(\pi^{+} \pi^{-}\right)$, the deviation of $s$ from the threshold value $4 m_{\pi^{+}}^{2}$ is of order $O\left(\alpha^{2}\right)$. The quantities $b_{+-}^{2}(s), b_{00}^{2}(s), b_{0,+-}^{2}(s), t$ and $u$ are of order $O\left(\alpha^{2}\right)$. The quantity $b_{0,00}^{2}$ of the $\pi^{0} \pi^{0}$ system at the same energy is of order $O\left(\Delta m_{\pi} / m_{\pi}\right)$.

\section{Wave equations of the $\pi^{+} \pi^{-}$and $\pi^{0} \pi^{0}$ systems}

In order to deal with the specific sectors of the $\pi^{+} \pi^{-}$and $\pi^{0} \pi^{0}$ systems, we have to enlarge the spaces of potentials and wave functions considered in Sec. 2. We introduce a two-component wave function $\Psi$ as:

$$
\Psi=\left(\begin{array}{c}
\Psi_{+-} \\
\Psi_{00}
\end{array}\right)
$$

and define the potential $\widetilde{V}$ in matrix form in the corresponding space:

$$
\widetilde{V}=\left(\begin{array}{cc}
V_{+-,+-} & V_{+-, 00} \\
V_{00,+-} & V_{00,00}
\end{array}\right)
$$

The constraint propagator $\widetilde{g}_{0}$ [Eqs. (2.2), (2.3) and (2.7)] is now:

$$
\left[\widetilde{g}_{0}\right]=\left(\begin{array}{cc}
\widetilde{g}_{0,+-} & 0 \\
0 & \frac{1}{2} \widetilde{g}_{0,00}
\end{array}\right), \quad\left[\widetilde{g}_{0}\right]^{-1}=\left(\begin{array}{cc}
\widetilde{g}_{0,+-}^{-1} & 0 \\
0 & 2 \widetilde{g}_{0,00}^{-1}
\end{array}\right),
$$

where the subscripts +- and 00 have been associated with $\widetilde{g}_{0}$, in order to take account, when necessary, of the specific free masses of the systems $\pi^{+} \pi^{-}$and $\pi^{0} \pi^{0}$ in formula (2.7). The factor $1 / 2$ in front of $\widetilde{g}_{0,00}$ has been introduced because of the identity of the particles in the corresponding sector.

Let $T_{+-,+-}, T_{+-, 00}, T_{00,+-}$ and $T_{00,00}($ with $\mathcal{M} \equiv-i T)$ designate the scattering amplitudes of the processes $\pi^{+} \pi^{-} \rightarrow \pi^{+} \pi^{-}, \pi^{0} \pi^{0} \rightarrow \pi^{+} \pi^{-}$, etc.. We use for our calculations the chiral effective lagrangian [3, 4, 21] in the $S U(2) \times S U(2)$ case. The scattering amplitude 
is obtained from the amputated four-point Green's function of pseudoscalar densities, multiplied by the corresponding wave function renormalization factors. We shall use for the field $U$ of the chiral effective lagrangian the representation

$$
U=\sigma+i \frac{\pi}{F_{0}} . \tau, \quad \sigma=\sqrt{1-\frac{\pi^{2}}{F_{0}^{2}}},
$$

where $\boldsymbol{\tau}$ are the Pauli matrices and $\boldsymbol{\pi}$ the pion fields. The pseudoscalar densities are defined as $P^{a}=i \bar{q} \gamma_{5} \tau^{a} q(a=1,2,3)$, where $q$ are the quark fields. Their Green's functions are obtained by deriving the generating functional with respect to the pseudoscalar sources. In the representation (3.4) and for the $O\left(p^{2}\right)$ lagrangian the pseudoscalar densities $P^{a}$ are proportional to the fields $\pi^{a}$ by the common constant factor $2 B_{0} F_{0}$. In the higher-order lagrangian terms more complicated differences arise and they should be taken into account. We shall draw Feynman diagrams with respect to the pion fields $\pi^{a}$.

In the strong interaction limit and in the absence of isospin breaking, the amplitudes $T$ above are related to the conventional amplitudes $A(s \mid t, u)$, etc., with the relations:

$$
\begin{aligned}
-i T_{+-,+-}^{s t r .} & =\mathcal{M}_{+-,+-}^{s t r .}=A(s \mid t, u)+A(t \mid s, u) \\
-i T_{+-, 00}^{s t r .} & =-i T_{00,+-}^{s t r .}=\mathcal{M}_{+-, 00}^{s t r .}=A(s \mid t, u) \\
-i T_{00,00}^{s t r .} & =\mathcal{M}_{00,00}^{s t r .}=A(s \mid t, u)+A(t \mid s, u)+A(u \mid t, s),
\end{aligned}
$$

and in terms of the isospin invariant amplitudes $T^{(I)}$, they are [5]:

$$
\begin{aligned}
T_{+-,+-}^{\text {str. }} & =\frac{1}{6} T^{(2)}+\frac{1}{2} T^{(1)}+\frac{1}{3} T^{(0)} \\
T_{+-, 00}^{\text {str. }} & =T_{00,+-}^{s t r .}=\frac{1}{3} T^{(0)}-\frac{1}{3} T^{(2)} \\
T_{00,00}^{\text {str. }} & =\frac{2}{3} T^{(2)}+\frac{1}{3} T^{(0)}
\end{aligned}
$$

The scattering lengths are defined as:

$$
-i T^{(I)}\left(s=4 m_{\pi}^{2}\right)=32 \pi a_{l=0}^{I}
$$

Equations (2.3) take now the explicit forms:

$$
\begin{aligned}
V_{+-,+-} & =\widetilde{T}_{+-,+-}+V_{+-,+-} \widetilde{g}_{0,+-} \widetilde{T}_{+-,+-}+\frac{1}{2} V_{+-, 00} \widetilde{g}_{0,00} \widetilde{T}_{00,+-}, \\
V_{+-, 00} & =\widetilde{T}_{+-, 00}+V_{+-,+-} \widetilde{g}_{0,+-} \widetilde{T}_{+-, 00}+\frac{1}{2} V_{+-, 00} \widetilde{g}_{0,00} \widetilde{T}_{00,00} \\
V_{00,+-} & =\widetilde{T}_{00,+-}+V_{00,+-} \widetilde{g}_{0,+-} \widetilde{T}_{+-,+-}+\frac{1}{2} V_{00,00} \widetilde{g}_{0,00} \widetilde{T}_{00,+-}, \\
V_{00,00} & =\widetilde{T}_{00,00}+V_{00,+-} \widetilde{g}_{0,+-} \widetilde{T}_{+-, 00}+\frac{1}{2} V_{00,00} \widetilde{g}_{0,00} \widetilde{T}_{00,00}
\end{aligned}
$$


We isolate from the potential $V_{+-,+-}$the Coulomb potential part [Eq. (2.8)]:

$$
V_{+-,+-}=V_{\text {Coul. }}+\bar{V}_{+-,+-} .
$$

The wave equations (2.2) then become:

$$
\begin{aligned}
-\widetilde{g}_{0,+-}^{-1} \Psi_{+-} & =\left(V_{\text {Coul. }}+\bar{V}_{+-,+-}\right) \Psi_{+-}+V_{+-, 00} \Psi_{00}, \\
-\widetilde{g}_{0,00}^{-1} \Psi_{00} & =\frac{1}{2} V_{00,+-} \Psi_{+-}+\frac{1}{2} V_{00,00} \Psi_{00} .
\end{aligned}
$$

These two wave equations are characterized by the same eigenvalue $P^{2}$. Whenever not specified, all potentials will be considered at the pionium ground state c.m. energy. At zeroth order (Coulomb potential only), it is:

$$
P_{0}=2 m_{\pi^{+}}-\frac{m_{\pi^{+}}}{4} \alpha^{2} .
$$

For this value of $P_{0}$, the operator $\widetilde{g}_{0,00}$ in Eq. (3.11) has values in the scattering region of the $\pi^{0} \pi^{0}$ system and therefore it gives rise to a scattering state for the wave function $\Psi_{00}$. The energy shift of the pionium is obtained by first eliminating $\Psi_{00}$ from Eq. (3.10) in terms of $\Psi_{+-}$through Eq. (3.11), using there the boundary condition that $\Psi_{00}$ is an outgoing wave due entirely to the presence of $\Psi_{+-}$.

The wave function $\Psi_{00}$ can be expressed in terms of $\Psi_{+-}$by iterating Eq. (3.11) with its last term (proportional to $V_{00,00}$ ). This iteration, where the dominant contribution comes from the strong interaction sector, when treated globally as a first-order perturbation, yields in the pionium decay width expression the unitarity factor [25, 27] $\left(1+(2 / 9)\left(\Delta m_{\pi} / m_{\pi}\right)\left(2 a_{0}^{2}+a_{0}^{0}\right)^{2}\right)^{-1}$; the correction term to one, being of the order of $10^{-4}$, will be neglected in the following. We thus obtain the wave equation:

$$
-\widetilde{g}_{0,+-}^{-1} \Psi_{+-}=\left[V_{\text {Coul } .}+\bar{V}_{+-,+-}-\frac{1}{2} V_{+-, 00,} \widetilde{g}_{0,00} V_{00,+-}\right] \Psi_{+-} .
$$

To the order of approximations we are working, the potentials $\bar{V}_{+-,+-}, V_{+-, 00}$ and $V_{00,+-}$ are, in $x$-space, three-dimensional delta-functions and hence they project all multiplicative quantities on their values at the origin. The value at the origin of the function $\widetilde{g}_{0,00}$ is calculated by Fourier transformation to momentum space and dimensional regularization. Designating by $\Delta m_{\pi}$ the pion mass difference [Eq. (1.2)], one finds (see Appendix A, Eq. (A.3)):

$$
\widetilde{g}_{0,00}(r=0)=\frac{i}{4 \pi} \sqrt{\Delta m_{\pi}\left(m_{\pi^{+}}+m_{\pi^{0}}\right)} .
$$

This term induces an imaginary part to the pionium energy. 


\section{Lowest-order formula with mass shift}

We shall determine a lowest-order expression for the pionium decay width with the inclusion in it of the main part of the pion mass shift. This formula will prove useful for the evaluation of the various types of correction. It is obtained by treating the last term of Eq. (3.13) in first-order of perturbation theory and by keeping in the potential $V_{00,+-}$ its dominant part, which comes essentially from the strong interactions. The expression of $V_{00,+-}$ [Eq. (3.8)] contains three terms. The second and third terms are the constraint diagram contributions, which will be considered below. The first term is the scattering amplitude. The latter can be split into two parts, the strong interaction part and the rest, which represents the contributions containing electromagnetic effects. Among the latter, there is one piece which plays a crucial role; it is the quark-photon (or massive hadron-photon) interaction term in the chiral limit, which is reponsible of the main part of the pion mass difference 443. It corresponds to the $O\left(e^{2} p^{0}\right)$ term of the chiral effective lagrangian with the expression $e^{2} C<Q U Q U^{\dagger}>$, where $C$ is an unknown constant and $Q$ is the quark charge matrix [44, 45]. Using for the field $U$ the representation (3.4), one finds:

$$
e^{2} C<Q U Q U^{\dagger}>=-\frac{2 e^{2} C}{F_{0}^{2}} \pi^{+} \pi^{-},
$$

which shows that this term induces for the charged pions the mass shift:

$$
\left(\Delta m_{\pi}^{2}\right)_{q \gamma}=2 e^{2} \frac{C}{F_{0}^{2}}
$$

which is nonvanishing in the chiral limit. Otherwise, it has no effect on the scattering amplitude in lowest order. (We emphasize that expression (1.1) of the latter is also valid off the mass-shell and does not depend of any mass-shell prescription.) Therefore, the quark-photon interaction term (4.1) acts essentially through insertions in the pion loop propagators, where the charged pion masses are replaced by their (almost) physical masses. Because the pionium lifetime evaluation is sensitive to the pion mass difference, it is natural to incorporate from the start the quark-photon interaction term (4.1) and its counterterms of the higher-order lagrangian in the strong interaction lagrangian. With this prescription, the amplitude $\mathcal{M}$ is split into two terms:

$$
\mathcal{M}=\mathcal{M}^{s t r .+q \gamma}+\mathcal{M}^{e m .}
$$


where $\mathcal{M}^{e m}$. contains all interference terms between electromagnetic and strong interaction effects, except the lowest-order quark-photon interaction term and its counterterms, which are included, together with the strong interaction terms, in $\mathcal{M}^{s t r . q \gamma}$.

We now turn to the evaluation of the two constraint diagrams corresponding to the last two terms of the expression of the potential $V_{00,+-}$ [Eq. (3.8)]. The evaluation of these diagrams is done by first considering the potentials and amplitudes $\widetilde{T}^{\text {str. }}$ at the tree level (Fig. 1) and calculating the loop integrals with the physical pion masses (cf. the comments above). The details of the calculations are presented in Appendix A. It is found that the terms in the amplitudes proportional to $t, u, b^{2}$ and $b_{0}^{2}$ yield, after integration, terms that are again of the same order and hence can be neglected. The leading terms are directly obtained by ignoring the above terms in the amplitudes and integrating over $\widetilde{g}_{0}$. One finds:

$$
\begin{aligned}
\widetilde{T}_{+-,+-} \widetilde{g}_{0,+-} \widetilde{T}_{+-,+-} & =-\frac{1}{4 \pi} \sqrt{-b_{0,+-}^{2}}\left(\widetilde{T}_{+-,+-}\right)^{2}, \\
\widetilde{T}_{+-, 00} \widetilde{g}_{0,00} \widetilde{T}_{00,+-} & =+\frac{i}{4 \pi} \sqrt{b_{0,00}^{2}} \widetilde{T}_{+-, 00} \widetilde{T}_{00,+-},
\end{aligned}
$$

(there are no integrations in the right-hand side,) and so forth for the other amplitudes, it being clear that $\widetilde{g}_{0,+-}$ yields a real contribution and $\widetilde{g}_{0,00}$ an imaginary one. By the very choice of the constraint propagators $\widetilde{g}_{0}$, the imaginary terms that arise from the constraint diagrams cancel similar terms coming from loop diagrams contained in the amplitudes $\widetilde{T}$ (the first terms of the right-hand sides of Eqs. (3.8)). Therefore, these amplitudes are replaced by their real parts. Concerning the real part of the constraint diagram, it is of order $O(\alpha)$ and has the opposite value of the $O(\alpha)$ part of the deviation of the scattering amplitude from the $\pi^{+} \pi^{-}$threshold down to the pionium energy; it comes from the finite non-analytic part of the unitarity loop, the polynomial parts giving only contributions of order $O\left(\alpha^{2}\right)$ to the above deviation. Hence, the real part of the constraint diagram shifts the real part of the scattering amplitude to its value at the $\pi^{+} \pi^{-}$threshold. We thus obtain:

$$
\begin{aligned}
V_{+-,+-}^{(0)} & =\mathcal{R} e \widetilde{T}_{+-,+-}^{(0)}\left(s=4 m_{\pi^{+}}^{2}\right), \\
V_{00,+-}^{(0)} & =\mathcal{R} e \widetilde{T}_{00,+-}^{(0)}\left(s=4 m_{\pi^{+}}^{2}\right), \\
V_{00,00}^{(0)} & =\mathcal{R} e \widetilde{T}_{00,00}^{(0)}\left(s=4 m_{\pi^{+}}^{2}\right) .
\end{aligned}
$$

The above property can also be generalized to the two-loop level of the strong inter- 
action amplitude. The details of the derivation are presented in Appendix B.

The part of the potential $V_{00,+-}$ that will contribute to the lowest-order expression of the decay width is then provided by the real part of $\mathcal{M}_{00,+-}^{\text {str. }+q \gamma}$ through $\mathcal{R} e \widetilde{T}_{00,+-}^{(0)}$. Defining

$$
P_{0}=P_{0 R}-i \frac{\Gamma}{2}
$$

one finds the modified lowest-order expression of the pionium decay width:

$$
\frac{1}{\bar{\tau}_{0}}=\bar{\Gamma}_{0}=\frac{1}{64 \pi m_{\pi^{+}}^{2}}\left(\mathcal{R} e \mathcal{M}_{00,+-}^{s t r+q \gamma}\right)^{2}\left|\psi_{+-}(0)\right|^{2} \sqrt{\frac{2 \Delta m_{\pi}}{m_{\pi^{+}}}\left(1-\frac{\Delta m_{\pi}}{2 m_{\pi^{+}}}\right)},
$$

where $\mathcal{R} e \mathcal{M}_{00,+-}^{\text {str. }+q \gamma}$ is calculated at the $\pi^{+} \pi^{-}$threshold. ( $\psi$ is the relative motion part of $\Psi$.) Furthermore, because the external particle momenta are subjected to the constraints $p_{1 L}=p_{2 L}=P_{L} / 2, p_{3 L}=p_{4 L}=P_{L} / 2$, with $P_{L}=2 m_{\pi^{+}}$and the kinetic energy operators $\left|p^{T 2}\right|$ and $\left|p^{T 2}\right|$ have, in the bound state, values of the order of magnitude of $O\left(\alpha^{2}\right)$, the mass-shell conditions in the above amplitude are:

$$
p_{1}^{2}=p_{2}^{2}=p_{3}^{2}=p_{4}^{2}=m_{\pi^{+}}^{2}, \quad s=4 m_{\pi^{+}}^{2}, \quad t=u=0 .
$$

With respect to the lowest-order formula (1.2), formula (4.7) contains two corrections. The first one, which has a kinematic origin, is included in the square-root term. The second one is included in $\mathcal{M}^{s t r .+q \gamma}$, where now, because of the pion mass difference, a modification occurs from the expression involving the scattering lengths calculated with the strong interaction amplitude.

In the remaining part of this paper we shall evaluate the $O(\alpha)$-corrections to the formula (4.7) as well as the modifications contained in $\mathcal{M}^{\text {str. }+q \gamma}$.

\section{Second-order perturbation theory}

In this section, we evaluate the effects coming from the second-order perturbation theory treatment of the strong interaction potential. At this order, it is the interference of the last two potential terms of Eq. (3.13) that contributes to the imaginary part of the energy. We have to distinguish here between the contributions of the discrete and continuum states of the pionium spectrum. We first consider the discrete spectrum contribution. 


\subsection{Contribution of the discrete spectrum}

Designating by $\psi_{+-, n}(n \geq 1)$ the zeroth order radial exitation wave functions of the ground state $[\psi$ is the relative motion part of $\Psi]$ and by $E_{n}$ the corresponding nonrelativistic energies, the shift in the decay width is:

$$
\begin{gathered}
(\Delta \Gamma)_{\text {discr. }}=-i \frac{2}{m_{\pi}} \widetilde{g}_{0,00}(r=0) V_{00,+-}^{2} \bar{V}_{+-,+-} \psi_{+-}^{2}(0) \\
\times \sum_{n=1}^{\infty} \frac{\psi_{+-, n}^{2}(0)}{m_{\pi}\left(E_{0}-E_{n}\right)} .
\end{gathered}
$$

Using the nonrelativistic formulas

$$
E_{n}=-\frac{m_{\pi} \alpha^{2}}{4(n+1)^{2}}, \quad \psi_{+-, n}^{2}(0)=\frac{m_{\pi}^{3} \alpha^{3}}{8 \pi(n+1)^{3}} \quad(n \geq 0),
$$

one finds:

$$
(\Delta \Gamma)_{\text {discr. }}=-\frac{\alpha}{4 \pi} m_{\pi} \bar{V}_{+-,+-} \Gamma_{0}=\frac{\alpha}{3}\left(2 a_{0}^{0}+a_{0}^{2}\right) \Gamma_{0},
$$

where in the last expression we have neglected the pion mass difference and introduced the strong interaction scattering lengths (3.7).

\subsection{Contribution of the continuous spectrum}

For the evaluation of the contribution of the continuum states, we introduce the nonrelativistic wavevector modulus $k=\sqrt{E m_{\pi}}$ and normalize wave functions as:

$$
\int d^{3} \mathbf{x} \psi_{k^{\prime}}^{*}(\mathbf{x}) \psi_{k}(\mathbf{x})=2 \pi \delta\left(k-k^{\prime}\right) .
$$

The shift in the decay width is then:

$$
(\Delta \Gamma)_{\text {cont. }}=-2 \Gamma_{0} \bar{V}_{+-,+-} \int \frac{d k}{2 \pi} \frac{\left|\psi_{k}(0)\right|^{2}}{\left(k^{2}+m_{\pi}^{2} \alpha^{2} / 4\right)},
$$

with [46]:

$$
\left|\psi_{k}(0)\right|^{2}=\frac{1}{4 \pi} \frac{4 \pi m_{\pi} \alpha k}{\left(1-\exp \left(-\pi m_{\pi} \alpha / k\right)\right)} .
$$

It is easily seen that $(\Delta \Gamma)_{\text {cont. }}$ diverges linearly in the ultra-violet region. In dimensional regularization, linear divergences being equivalent to zero, the divergence that survives in Eq. (5.5) is logarithmic:

$$
(\Delta \Gamma)_{\text {cont. }} \underset{k \rightarrow \infty}{\approx}-2 \Gamma_{0} \bar{V}_{+-,+-} \frac{m_{\pi} \alpha}{4 \pi} \ln \left(\frac{k}{m_{\pi}}\right) .
$$


The origin of this divergence is related to the singularity of the three-dimensional deltafunction that characterizes the potentials in $x$-space. In quantum mechanics, potentials that are proportional to three-dimensional delta-functions must be regularized through a renormalization of the coupling constant [47] or a self-adjoint extension of the hamiltonian [48. In both cases, an additional unknown parameter appears in the spectrum. On the other hand, a bound state equation, based on field theory, should not introduce new unknown parameters into the set of parameters already defined or fixed by the field theory itself. This is why one should expect the cancellation of the divergence (5.7) by some other terms present in the potential.

The constraint diagrams corresponding to the process $\pi^{+} \pi^{-} \rightarrow \pi^{0} \pi^{0}$ with one photon exchange and two loops (Fig. 2) play this role. They have overall logarithmic divergences that cancel the one appearing in $(\Delta \Gamma)_{\text {cont. }}$. Therefore, these two types of contribution should be considered together.

We now consider the contributions of the above constraint diagrams. At the vertex $\pi^{+} \pi^{-}-\pi^{0} \pi^{0}$, the amplitude that contributes is $A(s \mid t, u)$ [Eqs. (3.5) and (1.1)]; in lowest order, it depends only on the variable $s$ and therefore can be factored out of the integrals. At the vertex $\pi^{+} \pi^{-}-\pi^{+} \pi^{-}$, it is the amplitude $A(s \mid t, u)+A(t, \mid s, u)$ that contributes. However, the contribution of the variable $t$, present in $A(t \mid s, u)$, is of order $O\left(\alpha^{2}\right)$ when considered in the bound state domain (cf. also Appendix A); therefore, it can be omitted and the amplitude $A(s \mid t, u)+A(t \mid s, u)$ can also be factored out. We use for the potentials arising from the constraint diagrams the notation $V_{00,+-}^{C(n, m, p)}, C$ referring to "constraint", $n$ to the number of loops, $m$ to the number of exchanged photons and $p$ to the number of the constraint factors $\widetilde{g}_{0}$.

We first consider the constraint diagram of Fig. 2-c. The corresponding potential is (in the Feynman gauge):

$$
\begin{aligned}
V_{00,+-}^{C(2,1,2)}= & e^{2} \frac{i}{2 \sqrt{s}} V_{00,+-} \bar{V}_{+-,+-} \int \frac{d^{3} k^{T}}{(2 \pi)^{3}} \frac{d^{3} k^{\prime T}}{(2 \pi)^{3}} \\
& \times \frac{\left(2 p_{1}-k^{T}-k^{\prime T}\right) \cdot\left(2 p_{2}+k^{T}+k^{\prime T}\right)}{\left(\left(p_{1}-k^{\prime T}\right)^{2}-m_{\pi}^{2}+i \epsilon\right)\left(\left(p_{1}-k^{T}\right)^{2}-m_{\pi}^{2}+i \epsilon\right)} \frac{-i}{\left(k^{T}-k^{\prime T}\right)^{2}+i \epsilon} \\
\equiv & -i e^{2} V_{00,+-} V_{+-,+-} \int \frac{d^{3} k^{T}}{(2 \pi)^{3}} \frac{1}{\left(p_{1}-k^{T}\right)^{2}-m_{\pi}^{2}+i \epsilon} \\
& \times\left\{\left(4 p_{1} \cdot p_{2}+4 p^{T} \cdot k^{T}\right) F^{(1) C}\left(p_{1}, k^{T}\right)+4\left(p^{T}-k^{T}\right)^{\mu} F_{\mu}^{(1) C}\left(p_{1}, k^{T}\right)-R^{C}\left(p_{1}\right)\right\},
\end{aligned}
$$


with:

$$
\begin{aligned}
F^{(1) C}\left(p_{1}, k^{T}\right) & =\frac{i}{2 \sqrt{s}} \int \frac{d^{3} k^{\prime T}}{(2 \pi)^{3}} \frac{1}{\left(\left(p_{1}-k^{\prime T}\right)^{2}-m_{\pi}^{2}+i \epsilon\right)\left(\left(k^{T}-k^{T}\right)^{2}+i \epsilon\right)} \\
& =F^{(1) C}\left(p_{1}-k^{T}, 0\right) \\
& =\frac{i}{4 \sqrt{s}} \frac{1}{4 \pi} \frac{2}{\sqrt{-\left(p^{T}-k^{T}\right)^{2}}} \arctan \sqrt{\frac{-\left(p^{T}-k^{T}\right)^{2}}{-b_{0}^{2}(s)}}, \\
F_{\mu}^{(1) C}\left(p_{1}, k^{T}\right) & =\frac{i}{2 \sqrt{s}} \int \frac{d^{3} k^{\prime T}}{(2 \pi)^{3}} \frac{k_{\mu}^{\prime T}}{\left(\left(p_{1}-k^{\prime T}\right)^{2}-m_{\pi}^{2}+i \epsilon\right)\left(\left(k^{T}-k^{T}\right)^{2}+i \epsilon\right)} \\
& =k_{\mu}^{T} F^{(1) C}\left(p_{1}-k^{T}, 0\right)+F_{\mu}^{(1) C}\left(p_{1}-k^{T}, 0\right), \\
F_{\mu}^{(1) C}\left(p_{1}, 0\right) & =\frac{i}{8 s} p_{\mu}^{T} \sqrt{\frac{s}{b^{2}}}\left(\left(\frac{b^{2}-b_{0}^{2}}{b^{2}}\right) \arctan \sqrt{\frac{b^{2}}{-b_{0}^{2}}}-\sqrt{\frac{-b_{0}^{2}}{b^{2}}}\right), \\
\bar{R}^{C}\left(p_{1}\right)= & \frac{i}{2 \sqrt{s}} \int \frac{d^{3} k^{T}}{(2 \pi)^{3}} \frac{1}{\left(p_{1}-k^{T}\right)^{2}-m_{\pi}^{2}+i \epsilon}=-\frac{i}{2 \sqrt{s}} \frac{1}{4 \pi} \sqrt{-b_{0}^{2}} .
\end{aligned}
$$

[The integrals are finite in dimensional regularization. We have kept in $F_{\mu}^{(1) C}$ the leading terms only.]

In Eq. (5.8), it is only the term proportional to $p_{1} \cdot p_{2}$, through $p_{1 L} p_{2 L}$, that gives the surviving $O(\alpha)$ contribution. This observation makes also clear that the consideration of other covariant gauges would also yield the same leading term. Therefore, the subsequent results are gauge independent. One obtains:

$$
V_{00,+-}^{C(2,1,2)}=-m_{\pi} \alpha V_{00,+-} \bar{V}_{+-,+-} \int \frac{d^{3} k^{T}}{(2 \pi)^{3}} \frac{1}{m_{\pi}^{2}-p_{1 L}^{2}-k^{T 2}} \frac{1}{\sqrt{-k^{T 2}}} \arctan \sqrt{\frac{-k^{T 2}}{-b_{0}^{2}}} .
$$

We next consider the two constraint diagrams $a$ and $b$ of Fig. 2. Since the amplitude $A(s \mid t, u)+A(t \mid s, u)$ has been factored out of the integrals, the two diagrams give equal contributions. The four-dimensional loop corresponds to the electromagnetic radiative correction of the scalar vertex. Its expression will be calculated in Sec. 6 (and Appendix D). It contains, among other terms, a term that is the opposite of the function $F^{(1) C}$ [Eqs. (5.8)-(5.9)] we calculated above. [It is the first term in the expression of $F^{(1)}$ in Eq. (D.11), where $b^{2} \equiv-p^{T 2}$ should now be replaced by $-\left(p^{T}-k^{T}\right)^{2}$.] Its other terms contribute to nonleading effects in $\alpha$.

Therefore, the contribution resulting from the sum of the two diagrams (a) and (b) of Fig. 2 is:

$$
V_{00,+-}^{C(2,1,1)}=-2 V_{00,+-}^{C(2,1,2)} .
$$


The contribution to the shift in the decay width resulting from the three constraint diagrams of Fig. 2 is then:

$$
(\Delta \Gamma)^{C(2,1,1+2)}=2 m_{\pi} \alpha \Gamma_{0} \bar{V}_{+-,+-} \int \frac{d^{3} \mathbf{k}}{(2 \pi)^{3}} \frac{1}{\left(\mathbf{k}^{\mathbf{2}}+m_{\pi}^{2} \alpha^{2} / 4\right)} \frac{1}{k} \arctan \left(\frac{2 k}{m_{\pi} \alpha}\right),
$$

where we have replaced $-b_{0}^{2} \equiv m_{\pi}^{2}-p_{1 L}^{2}$ by its eigenvalue $m_{\pi}^{2} \alpha^{2} / 4$.

$(\Delta \Gamma)^{C}$ has an ultra-violet divergence that is the opposite of that of $(\Delta \Gamma)_{\text {cont. }}[\mathrm{Eq}$. (5.7)]. Therefore, the sum $(\Delta \Gamma)^{C}+(\Delta \Gamma)_{\text {cont. }}$ is finite. One obtains:

$$
\begin{aligned}
(\Delta \Gamma)^{C(2,1,1+2)}+(\Delta \Gamma)_{\text {cont. }}= & 2 m_{\pi} \alpha \Gamma_{0} \bar{V}_{+-,+-} \frac{1}{4 \pi} \\
& \times \int_{0}^{\infty} d x\left(\frac{x}{x^{2}+1}\right)\left[\frac{2}{\pi} \arctan x+\frac{x}{\pi}-\frac{2}{1-e^{-2 \pi / x}}\right] \\
\simeq & -1.7 m_{\pi} \frac{\alpha}{2 \pi} \Gamma_{0} \bar{V}_{+-,+-}=1.1 \alpha\left(2 a_{0}^{0}+a_{0}^{2}\right) \Gamma_{0} .
\end{aligned}
$$

[We have subtracted from $(\Delta \Gamma)_{\text {cont. }}$ its linear divergence, which is null in dimensional regularization.] The constraint diagrams have played the role of an effective cut-off of the divergence of $(\Delta \Gamma)_{\text {cont. }}$. Had we cut the integral in Eq. (5.5) at the value $k=m_{\pi} \alpha$, we would have found a result close to that of Eq. (5.15).

In the previous cancellation mechanism of divergences we used dimensional regularization and hence ignored linear divergences. The latter are present in conventional calculations of integrals and for such type of calculation they should be taken into account. The point is that in this case the constraint diagrams themselves are linearly divergent and these divergences should be isolated and grouped essentially with those coming from second-order perturbation theory. It is not difficult to show that the linear divergences cancel out among themselves and the physical results are not affected by their presence. Details of the derivation are presented in Appendix C. The cancellation mechanism of divergences is therefore regularization scheme independent.

The total amount of the strong interaction corrections is given by the sum of $(\Delta \Gamma)_{\text {discr. }}$. [Eq. (5.3)] and $(\Delta \Gamma)^{C(2,1,1+2)}+(\Delta \Gamma)_{\text {cont. }}[$ Eq. (5.15)]. One obtains:

$$
\begin{aligned}
(\Delta \Gamma)_{\text {str. }} & =(\Delta \Gamma)_{\text {discr. }}+\left((\Delta \Gamma)_{\text {cont. }}+(\Delta \Gamma)^{C(2,1,1+2)}\right) \\
& =1.5 \alpha\left(2 a_{0}^{0}+a_{0}^{2}\right) \Gamma_{0}
\end{aligned}
$$

The value of $(\Delta \Gamma)_{\text {str. }}$ depends on the values of the scattering lengths and hence also of the quark condensate parameter $2 \hat{m} B_{0}$ [Eq. (1.1)]. In the standard case $\left(2 \hat{m} B_{0} \simeq m_{\pi}^{2}\right)[3$, 
13] one has $1.5\left(2 a_{0}^{0}+a_{0}^{2}\right) \simeq 0.55$. In the other extreme case $\left(2 \hat{m} B_{0}=0\right)$, one has $1.5\left(2 a_{0}^{0}+a_{0}^{2}\right)$ $\simeq 0.9$ [22, 23]. Therefore, in all cases the correction $\left(\Delta \Gamma / \Gamma_{0}\right)_{s t r}$. is bounded by the values:

$$
\left(\frac{\Delta \Gamma}{\Gamma_{0}}\right)_{s t r .}= \begin{cases}0.55 \alpha=0.004 & \left(2 \hat{m} B_{0}=m_{\pi}^{2}\right), \\ 0.9 \alpha=0.0065 & \left(2 \hat{m} B_{0}=0\right) .\end{cases}
$$

\section{Electromagnetic radiative corrections}

In this section we calculate the electromagnetic radiative corrections arising fom pionphoton interaction and contributing to the amplitude $\mathcal{M}^{\mathrm{em}}$. of the decomposition (4.3).

We first evaluate the unrenormalized quantities. The lagrangian corresponding to the pion-photon interaction is obtained by appropriately incorporating in the external vector current of the chiral effective lagrangian the photon field. Using for the field $U$ the representation (3.4), the corresponding lagrangian becomes in lowest order:

$$
\begin{aligned}
\mathcal{L}= & \frac{1}{2} \partial_{\mu} \pi^{0} \partial^{\mu} \pi^{0}+\left(\partial_{\mu}+i e A_{\mu}\right) \pi^{+}\left(\partial^{\mu}-i e A^{\mu}\right) \pi^{-} \\
& -\frac{1}{2} m_{\pi}^{2}\left(\pi^{0} \pi^{0}+2 \pi^{+} \pi^{-}\right)-\frac{1}{4} F_{\mu \nu} F^{\mu \nu}-\frac{1}{2(1-\xi)}(\partial . A)^{2} \\
& +\frac{1}{8 F_{0}^{2}} \partial_{\mu}\left(\pi^{02}+2 \pi^{+} \pi^{-}\right) \partial^{\mu}\left(\pi^{02}+2 \pi^{+} \pi^{-}\right) \\
& -\frac{1}{8 F_{0}^{2}}\left(2 \hat{m} B_{0}\right)\left(\pi^{04}+4 \pi^{+} \pi^{-} \pi^{0} \pi^{0}+4 \pi^{+2} \pi^{-2}\right),
\end{aligned}
$$

$2 \hat{m} B_{0}$ being defined in Eq. (1.1).

We begin with the self-energy correction, which is (photon tadpole contributions are null in dimensional regularization):

$$
\begin{aligned}
-i \Sigma(p) & =-e^{2} \int \frac{d^{4} k}{(2 \pi)^{4}} \frac{\left((2 p-k)^{2}-\xi[(2 p-k) \cdot k]^{2} / k^{2}\right)}{\left((p-k)^{2}-m_{\pi}^{2}+i \epsilon\right)\left(k^{2}+i \epsilon\right)} \\
& \equiv-e^{2}\left[4 p^{2} R^{(1)}(p)+(1-\xi) T(p)-4(1-\xi) p^{\mu} R_{\mu}^{(1)}(p)-4 \xi p^{\mu} p^{\nu} R_{\mu \nu}^{(2)}(p)\right],
\end{aligned}
$$

where $\xi$ represents the gauge parameter. The definitions and expressions of the functions $R$ and $T$ are presented in Appendix D. The expression of the unrenormalized self-energy is given in Eq. (D.8). The unrenormalized Green's function of the charged pseudoscalar density becomes:

$$
G^{+}(p)=\frac{i\left(2 B_{0} F_{0}\right)^{2}}{p^{2}-\left(m_{\pi}^{2}+\left(\Delta m_{\pi}^{2}\right)_{\pi \gamma}\right)+i \epsilon}\left(1-\Delta Z^{+}(p)\right),
$$


with:

$$
\begin{aligned}
\left(\Delta m_{\pi^{+}}^{2}\right)_{\pi \gamma} & =\left(\frac{3 e^{2}}{\varepsilon}+\frac{7 \alpha}{4 \pi}\right) m_{\pi}^{2}, \\
-\Delta Z^{+}(p) & =(2+\xi) \frac{e^{2}}{\varepsilon}+\frac{\alpha}{\pi}\left(1+\left(1+\frac{\xi}{2}\right) \ln \left(\frac{m_{\pi}^{2}}{\lambda^{2}}\right)+\frac{1}{2}\left(1+\frac{\xi}{2}\right) \frac{\lambda^{2}}{p^{2}} \ln \left(\frac{m_{\pi}^{2}}{\lambda^{2}}\right)\right),
\end{aligned}
$$

$\varepsilon$ and $\lambda^{2}$ being defined in Eqs. (D.6) and (D.7), respectively.

We next pass to the vertex function (Fig. 3a). Omitting from its definition the $\pi^{+} \pi^{-} \rightarrow \pi^{0} \pi^{0}$ amplitude $A(s \mid t, u)$, which at the tree level does not depend on $t$ and $u$ [Eq. (1.1)], it is:

$$
\begin{aligned}
\Lambda\left(p_{1}, p_{2}\right)= & i e^{2} \int \frac{d^{4} k}{(2 \pi)^{4}} \frac{\left(\left(2 p_{1}-k\right) \cdot\left(2 p_{2}+k\right)-\xi\left(2 p_{1}-k\right) \cdot k\left(2 p_{2}+k\right) \cdot k / k^{2}\right)}{\left(\left(p_{1}-k\right)^{2}-m_{\pi}^{2}+i \epsilon\right)\left(\left(p_{2}+k\right)^{2}-m_{\pi}^{2}+i \epsilon\right)\left(k^{2}+i \epsilon\right)} \\
\equiv & i e^{2}\left[-R^{(1)}\left(p_{1}\right)+\left(4 p_{1} \cdot p_{2}+p_{2}^{2}-m_{\pi}^{2}\right) F^{(1)}\left(p_{1}, p_{2}, 0\right)+2 p_{1}^{\mu} F_{\mu}^{(1)}\left(p_{1}, p_{2}, 0\right)\right] \\
& -i e^{2} \xi\left[-p_{1}^{\mu} \frac{\partial}{\partial p_{1}^{\mu}} R^{(1)}\left(p_{1}\right)+\left(m_{\pi}^{2}-p_{2}^{2}\right) 2 p_{1}^{\mu} F_{\mu}^{(2)}\left(p_{1}, p_{2}, 0\right)\right. \\
& \left.\quad-2 p_{2}^{\mu} F_{\mu}^{(1)}\left(p_{1}, p_{2}, 0\right)-\bar{R}\left(p_{1}, p_{2}\right)\right],
\end{aligned}
$$

with the definitions and expressions of the functions $R, \bar{R}$ and $F$ given in Appendix D.

To this order of approximation, the strong vertex part of $\Lambda$ is a scalar vertex and therefore $\Lambda$ satisfies an identity typical of mass operators, obtained by differentiation with respect to $m_{\pi}^{2}[49]$. It reads:

$$
\Lambda\left(p_{1},-p_{1}\right)=\frac{\partial \Sigma\left(p_{1}\right)}{\partial m_{\pi}^{2}}
$$

The unrenormalized $\Lambda$ at leading orders is given in Eq. (D.19).

In passing to the scattering amplitude, one must associate with the vertex function the wave function renormalization factors of the external particles (the square-root of $\left(1-\Delta Z^{+}\right)$of Eq. (6.3)). This leads to the combination:

$$
\Lambda\left(p_{1}, p_{2}\right)-\frac{1}{2} \Delta Z^{+}\left(p_{1}\right)-\frac{1}{2} \Delta Z^{+}\left(p_{2}\right)=\left(\frac{3 e^{2}}{\varepsilon}-\frac{\alpha}{2 \pi}\right)+\frac{\alpha}{2} \frac{4 p_{1} \cdot p_{2}}{s} \sqrt{\frac{s}{b^{2}}} \arctan \sqrt{\frac{b^{2}}{-b_{0}^{2}}} .
$$

[We have kept dominant terms only.] Notice that the gauge parameter and the logarithmic functions have been cancelled out.

With the above quantity we must associate the contribution of the constraint diagram arising from the interference term between the strong interaction amplitude and onephoton exchange diagram (Fig. 3b):

$$
\Lambda^{C}\left(p_{1}, p_{2}\right)=i e^{2} \frac{i}{2 \sqrt{s}} \int \frac{d^{3} k^{T}}{(2 \pi)^{3}} \frac{\left(\left(2 p_{1}-k^{T}\right) \cdot\left(2 p_{2}+k^{T}\right)-\xi\left(2 p_{1}-k^{T}\right) \cdot k^{T}\left(2 p_{2}+k^{T}\right) / k^{T 2}\right)}{\left(\left(p_{1}-k^{T}\right)^{2}-m_{\pi}^{2}+i \epsilon\right)\left(k^{T 2}+i \epsilon\right)}
$$




$$
\begin{aligned}
= & i e^{2}\left[-\bar{R}^{C}\left(p_{1}\right)+4 p_{1} \cdot p_{2} F^{(1) C}\left(p_{1}, 0\right)+4 p^{T \mu} F_{\mu}^{(1) C}\left(p_{1}, 0\right)\right] \\
& -i e^{2} \xi\left[2 \lambda^{2} p^{T \mu} F_{\mu}^{(2) C}\left(p_{1}, 0\right)+2 p^{T \mu} F_{\mu}^{(1) C}\left(p_{1}, 0\right)-\bar{R}^{C}\left(p_{1}\right)\right],
\end{aligned}
$$

the definitions and expressions of the functions $\bar{R}^{C}$ and $F^{C}$ being given in Eqs. (5.9)-(5.11) and Appendix D. One finds:

$$
\Lambda^{C}\left(p_{1}, p_{2}\right)=-\frac{\alpha}{2} \frac{4 p_{1} \cdot p_{2}}{s} \sqrt{\frac{s}{b^{2}}} \arctan \sqrt{\frac{b^{2}}{-b_{0}^{2}}} .
$$

Adding this term to the quantity in Eq. (6.8) yields the net effect of the unrenormalized radiative corrections on the potential $V_{00,+-}($ relative to 1$)$ :

$$
\Lambda\left(p_{1}, p_{2}\right)-\frac{1}{2} \Delta Z^{+}\left(p_{1}\right)-\frac{1}{2} \Delta Z^{+}\left(p_{2}\right)+\Lambda^{C}\left(p_{1}, p_{2}\right)=\left(\frac{3 e^{2}}{\varepsilon}-\frac{\alpha}{2 \pi}\right)
$$

which is a gauge invariant quantity. On the other hand, the constraint diagram has cancelled the term proportional to the arctan function present in $\Lambda$, which otherwise would give a contribution of order $O\left(\alpha^{0}\right)$.

We next consider the contributions of the $O\left(e^{2} p^{2}\right)$ counterterms present in the chiral effective lagrangian. The complete expression of the corresponding $S U(3) \times S U(3)$ lagrangian in the Feynman gauge (in the standard scheme, to which we stick henceforth) is given in Ref. [45], where the coefficients of the various terms are designated by $K_{i}$ $(i=1, \ldots, 17)$. These terms are of two categories. Those which are counterterms to the $O\left(e^{2} p^{0}\right)$ lagrangian (4.1) and the infinite parts of which are proportional to $C$, and those which are counterterms to the radiative corrections of the pion-photon interaction. Since the $\left(O\left(e^{2} p^{0}\right)\right.$ term is included in the lagrangian contributing to the amplitude $\mathcal{M}^{\text {str. }+q \gamma}$, so must be done with the corresponding counterterms. Therefore we have to select from the $O\left(e^{2} p^{2}\right)$ lagrangian those terms the infinite parts of which are not proportional to the constant $C$.

The $O\left(e^{2} p^{2}\right)$ lagrangian in the $S U(2) \times S U(2)$ case was recently presented in Ref. [50] (in the Feynman gauge). Since we are treating in the present work the $S U(2)$ case, we use for our subsequent calculations the latter lagrangian, indicating, when necessary, the correspondence between the $S U(2)$ and $S U(3)$ coefficients. The relevant part of this lagrangian for our problem is (in standard notations):

$$
\begin{aligned}
\mathcal{L}^{\left(e^{2} p^{2}\right)=F_{0}^{2}}\{ & \left\{k_{2}<Q U Q U^{\dagger}><\partial_{\mu} U \partial^{\mu} U^{\dagger}>\right. \\
& +k_{3}\left(<U^{\dagger} \partial_{\mu} U Q><U^{\dagger} \partial^{\mu} U Q>+<\partial_{\mu} U U^{\dagger} Q><\partial^{\mu} U U^{\dagger} Q>\right)
\end{aligned}
$$




$$
\begin{aligned}
& +k_{4}<U^{\dagger} \partial_{\mu} U Q><\partial^{\mu} U U^{\dagger} Q>+k_{7}<Q U Q U^{\dagger}><\chi U^{\dagger}+U \chi^{\dagger}> \\
& +k_{8}<\left(U^{\dagger} \chi-\chi^{\dagger} U\right)\left(U^{\dagger} Q U Q-Q U^{\dagger} Q U\right)> \\
& \left.+k_{10}<Q^{2}><\partial_{\mu} U \partial^{\mu} U^{\dagger}>+k_{11}<Q^{2}><\chi U^{\dagger}+U \chi^{\dagger}>\right\}
\end{aligned}
$$

where $Q$ is the quark charge matrix. The coefficients $k$ have the following decompositions:

$$
k_{i}=\kappa_{i} \bar{\lambda}+k_{i}^{r}(\mu)
$$

where $\bar{\lambda}$ has a pole in $d=4$ dimensions,

$$
\bar{\lambda}=\frac{1}{16 \pi^{2}}\left(\frac{1}{d-4}-\frac{1}{2}\left(\ln (4 \pi)+\Gamma^{\prime}(1)+1\right)\right),
$$

and the coefficients $\kappa$ have the valuesf:

$$
\begin{array}{llll}
\kappa_{2}=2 Z, & \kappa_{3}=-\frac{3}{4}, & \kappa_{4}=-2 Z, & \kappa_{7}=\frac{1}{4}+2 Z, \\
\kappa_{8}=\frac{1}{8}-Z, & \kappa_{10}=-\frac{27}{20}-\frac{Z}{5}, & \kappa_{11}=-\frac{1}{4}-\frac{Z}{5}, & Z=\frac{C}{F_{0}^{4} .}
\end{array}
$$

The $\mu$-dependence of the finite part $k_{i}^{r}(\mu)$ of $k_{i}$ is fixed by the prescription that its sum with the $\ln \left(\mu^{-2}\right)$ term of the corresponding loop diagrams be $\mu$-independent; the corresponding multiplicative coefficient is $\kappa_{i} /\left(32 \pi^{2}\right)$. The lagrangian (6.12), together with the coefficients (6.15), is associated with the $S U(2) \times S U(2)$ version of the chiral effective lagrangian of Ref. [3], in which the multiplicative factors have been arranged so that the coefficients $l_{i}$ keep the same values as those of the $O(4)$ version [Eqs. (7.1)-(7.3)].

The lagrangian (6.12) takes the following form in the representation (3.4), keeping only pion fields that contribute to the scattering problem to the present approximation:

$$
\begin{aligned}
\mathcal{L}^{\left(e^{2} p^{2}\right)=} & e^{2}\left(k_{2}+\frac{10}{9}\left(\frac{k_{2}}{10}+k_{10}\right)\right)\left[\partial_{\mu} \pi^{0} \partial^{\mu} \pi^{0}+2 \partial_{\mu} \pi^{+} \partial^{\mu} \pi^{-}\right. \\
& \left.\quad+\frac{1}{F_{0}^{2}}\left(\frac{1}{2} \partial_{\mu} \pi^{02}+\partial_{\mu}\left(\pi^{+} \pi^{-}\right)\right)\left(\frac{1}{2} \partial^{\mu} \pi^{02}+\partial^{\mu}\left(\pi^{+} \pi^{-}\right)\right)\right] \\
& -\frac{4 e^{2}}{F_{0}^{2}} k_{2} \pi^{+} \pi^{-}\left(\partial_{\mu} \pi^{0} \partial^{\mu} \pi^{0}+2 \partial_{\mu} \pi^{+} \partial^{\mu} \pi^{-}\right) \\
+ & \frac{e^{2}}{F_{0}^{2}}\left(2 k_{3}-k_{4}\right)\left(\pi^{+} \stackrel{\leftrightarrow}{\partial_{\mu}} \pi^{-}\right)\left(\pi^{+} \stackrel{\leftrightarrow}{\partial^{\mu}} \pi^{-}\right)-\frac{e^{2}}{F_{0}^{2}}\left(2 k_{3}+k_{4}\right)\left[F_{0}^{2} \partial_{\mu} \pi^{0} \partial^{\mu} \pi^{0}\right. \\
& \left.-\pi^{02} \partial_{\mu} \pi^{0} \partial^{\mu} \pi^{0}-2 \pi^{+} \pi^{-} \partial_{\mu} \pi^{0} \partial^{\mu} \pi^{0}+\frac{1}{2} \partial_{\mu} \pi^{02} \partial^{\mu} \pi^{02}+\partial_{\mu}\left(\pi^{02}\right) \partial^{\mu}\left(\pi^{+} \pi^{-}\right)\right] \\
& -\frac{5 e^{2}}{9}\left(k_{7}+k_{11}\right) 8 \hat{m} B_{0}\left[\frac{1}{2} \pi^{02}+\pi^{+} \pi^{-}+\frac{1}{8 F_{0}^{2}}\left(\pi^{02}+2 \pi^{+} \pi^{-}\right)^{2}\right] \\
& -\frac{2 e^{2}}{F_{0}^{2}}\left(\frac{1}{4}\left(k_{7}-2 k_{8}\right)+\frac{3}{4}\left(k_{7}+2 k_{8}\right)\right) 8 \hat{m} B_{0}\left[F_{0}^{2} \pi^{+} \pi^{-}-\pi^{+} \pi^{-}\left(\frac{1}{2} \pi^{02}+\pi^{+} \pi^{-}\right)\right] .
\end{aligned}
$$

\footnotetext{
${ }^{4}$ We thank M. Knecht for pointing out to us the presence of the factor $-Z$ in $\kappa_{8}$.
} 
The coefficients $\left(k_{2} / 10+k_{10}\right), k_{3}$ and $\left(k_{7}+2 k_{8}\right)$ contribute, at least concerning their infinite parts, only to the pion-photon interaction effects, while the coefficients $k_{2}, k_{4},\left(k_{7}+k_{11}\right)$ and $\left(k_{7}-2 k_{8}\right)$ contribute to the quark-photon interaction effects (insertions of the mass term (4.1)). We shall admit that this separation also holds for their finite parts.

The $O\left(e^{2} p^{2}\right)$ lagrangian (6.12) introduces new terms into the relationships between the pseudoscalar densities and the pion fields. These are:

$$
P^{a}=2 B_{0} F_{0}\left(1+\frac{20 e^{2}}{9}\left(k_{7}+k_{11}\right)+4 e^{2} k_{8} \delta_{a \pm}-2 e^{2}\left(\left(k_{7}-2 k_{8}\right)+3\left(k_{7}+2 k_{8}\right)\right) \frac{\pi^{+} \pi^{-}}{F_{0}^{2}}\right) \pi^{a},
$$

where $a=0,+,-$. The three-pion term yields additional diagrams for the scattering amplitude, which, however, contribute only off the mass shell. The term proportional to the coefficient $k_{8}$ alone contributes to both the pion-photon and quark-photon interaction effects. For this reason we shall separate this coefficient into two parts by writing, with an obvious notation, $k_{8}=k_{8}^{\pi \gamma}+k_{8}^{q \gamma}$. Although this separation is ambiguous for the corresponding finite parts, it will not show up in physical quantities.

To obtain the renormalized Green's functions of the pseudoscalar densities due to pion-photon interaction, we select among the above expreesions the quantities proportional to the relevant coefficients. Furthermore, since we are considering here on-mass shell expressions, the three-pion term of Eq. (6.17) can be discarded. One finds (in the Feynman gauge) the expressions:

$$
\begin{aligned}
G^{0}(p) & =\frac{i\left(2 B_{0} F_{0}\right)^{2}\left(1-\Delta Z^{0}\right)}{p^{2}-\left(m_{\pi}^{2}+\left(\Delta m_{\pi^{0}}^{2}\right)_{\pi \gamma}\right)+i \epsilon}, \\
-\Delta Z^{0} & =-\frac{2 e^{2}}{9}\left(k_{2}^{r}+10 k_{10}^{r}\right)+4 e^{2} k_{3}^{r}, \\
\left(\Delta m_{\pi^{0}}^{2}\right)_{\pi \gamma} & =m_{\pi}^{2}\left[-\frac{2 e^{2}}{9}\left(k_{2}^{r}+10 k_{10}^{r}\right)+4 e^{2} k_{3}^{r}\right] \\
G^{+}(p) & =\frac{i\left(2 B_{0} F_{0}\right)^{2}\left(1-\Delta Z^{+}\right)}{p^{2}-\left(m_{\pi}^{2}+\left(\Delta m_{\pi^{+}}^{2}\right)_{\pi \gamma}\right)+i \epsilon}, \\
-\Delta Z^{+} & =\frac{\alpha}{\pi}+\frac{\alpha}{\pi} \ln \left(\frac{m_{\pi}^{2}}{\lambda^{2}}\right)+\frac{\alpha}{2 \pi} \frac{\lambda^{2}}{p^{2}} \ln \left(\frac{m_{\pi}^{2}}{\lambda^{2}}-\frac{\alpha}{2 \pi}\left(\ln \left(\frac{m_{\pi}^{2}}{\mu^{2}}\right)+1\right)\right. \\
\left(\Delta m_{\pi^{+}}^{2}\right)_{\pi \gamma} & =m_{\pi}^{2}\left[\frac{7 \alpha}{4 \pi}-\frac{3 \alpha}{4 \pi}\left(\ln \left(\frac{m_{\pi}^{2}}{\mu^{2}}\right)+1\right)-\frac{2 e^{2}}{9}\left(k_{2}^{r}+10 k_{10}^{r}\right)+6 e^{2}\left(k_{7}^{r}+2 k_{8}^{r}\right)\right] .
\end{aligned}
$$


The difference between the charged and neutral pion masses due to pion-photon interaction is:

$$
\begin{aligned}
\left(\Delta m_{\pi}^{2}\right)_{\pi \gamma} & =\left(\Delta m_{\pi^{+}}^{2}\right)_{\pi \gamma}-\left(\Delta m_{\pi^{0}}^{2}\right)_{\pi \gamma} \\
& =\left[\frac{7 \alpha}{4 \pi}-\frac{3 \alpha}{4 \pi}\left(\ln \left(\frac{m_{\pi}^{2}}{\mu^{2}}\right)+1\right)-4 e^{2} k_{3}^{r}+6 e^{2}\left(k_{7}^{r}+2 k_{8}^{r}\right)\right] m_{\pi}^{2}
\end{aligned}
$$

To obtain the renormalized scattering amplitude of the process $\pi^{+} \pi^{-} \rightarrow \pi^{0} \pi^{0}$, we consider the sum of the unrenormalized four-point vertex function and of the corresponding constraint diagram contribution:

$$
\left[W_{00,+-}+W_{00,+-}^{C(1,1,1)}\right]_{\pi \gamma}=\left(2 B_{0} F_{0}\right)^{-4}\left(1+4 e^{2} k_{8}^{\pi \gamma}\right)^{-2} A(s \mid t, u)\left(1+\Lambda+\Lambda^{C}\right),
$$

where $A(s \mid t, u)$ is defined in Eq. (1.1), $\Lambda$ in Eq. (D.19) (taken here in the Feynman gauge) and $\Lambda^{C}$ in Eq. (6.10); the other multiplicative factors come from the differences between the pseudoscalar densities and the pion fields [Eqs. 6.17)]. The lagrangian (6.16) provides the following counterterms:

$$
\begin{array}{r}
\left(W_{00,+-}^{\left(e^{2} p^{2}\right)}\right)_{\pi \gamma}=\left(2 B_{0} F_{0}\right)^{-4} \frac{e^{2}}{F_{0}^{2}}\left[\left(\frac{2}{9}\left(k_{2}+10 k_{10}\right)-8 k_{3}\right) s\right. \\
\left.+8\left(k_{3}+\frac{3}{4}\left(k_{7}+2 k_{8}\right)\right) 2 \hat{m} B_{0}\right] .
\end{array}
$$

These remove the divergences of the unrenormalized vertex (6.21). The sum of the two quantities is then multiplied by the renormalized wave function renormalization factors $\left(2 B_{0} F_{0}\right)^{4}\left(1-\Delta Z^{0} / 2\right)^{2}\left(1-\Delta Z^{+} / 2\right)^{2}$ [Eqs. (6.18)-(6.19]). We also incorporate in the mass term of the lowest-order amplitude (1.1) the neutral pion mass shift (6.18). The renormalized scattering amplitude, together with the constraint diagram contribution, is then:

$$
\begin{aligned}
{\left[\mathcal{M}_{00,+-}+\mathcal{M}_{00,+-}^{C(1,1,1)}\right]_{\pi \gamma}=} & \frac{\left(s-m_{\pi^{0}}^{2}\right)}{F_{0}^{2}}\left[1-\frac{\alpha}{2 \pi}-\frac{3 \alpha}{4 \pi}\left(\ln \left(\frac{m_{\pi}^{2}}{\mu^{2}}\right)+1\right)-4 e^{2} k_{3}^{r}\right. \\
& \left.-\frac{2 e^{2}}{9}\left(k_{2}^{r}+10 k_{10}^{r}\right)\right]+2 e^{2} \frac{m_{\pi}^{2}}{F_{0}^{2}}\left[2 e^{2} k_{3}^{r}+3 e^{2}\left(k_{7}^{r}+2 k_{8}^{r}\right)\right] .
\end{aligned}
$$

The corresponding modification of the decay width is:

$$
(\Delta \Gamma)_{\pi \gamma}=\left[-\frac{\alpha}{\pi}-\frac{3 \alpha}{2 \pi}\left(\ln \left(\frac{m_{\pi}^{2}}{\mu^{2}}\right)+1\right)-\frac{4 e^{2}}{9}\left(k_{2}^{r}+10 k_{10}^{r}\right)-\frac{16 e^{2}}{3} k_{3}^{r}+4 e^{2}\left(k_{7}^{r}+2 k_{8}^{r}\right)\right] \Gamma_{0} .
$$


To estimate the correction (6.24) one needs to know the values of the coefficients $k^{r}$. At this time they are not yet well established, but several evaluations with different methods exist and can be used to have an idea of the order of magnitude and sign of the correction. The correspondence of the above coefficients $k$ with the $S U(3)$ coefficients $K$ is (valid also for the finite parts):

$$
\begin{aligned}
& k_{2}+10 k_{10}=12 K_{1}+10 K_{5}, \quad k_{3}=K_{3}, \\
& \frac{3}{4}\left(k_{7}+2 k_{8}\right)=-\frac{3}{2} K_{8}+K_{10}+K_{11} .
\end{aligned}
$$

In ref [51], the $K^{r}$ 's have been evaluated using a resonance model for the saturation of sum rules. The corresponding values are in units of $10^{-3}$ and at the scale $\mu=m_{\rho}$ : $K_{1}^{r}=-6.4, K_{3}^{r}=6.4, K_{5}^{r}=19.9, K_{8}^{r}=K_{9}^{r}=K_{10}^{r}=0, K_{11}=0.6$.

In Ref. [52] values of some of the $K^{r}$ 's have been evaluated with the aid of the $1 / N_{C^{-}}$ expansion method, the Extended Nambu-Jona-Lasinio model and perturbative QCD. The results are not completely compatible with those of Ref. [51. In particular it is found (at similar scales and in units of $10^{-3}$ ): $K_{9}^{r}=-1.3, K_{10}^{r}=4.0$; the other results involve $K^{r}$ 's with even indices. Estimates of $K_{10}^{r}$ and $K_{11}^{r}$ have been presented in Ref. [53 on the basis of sum rule analysis. The value of $K_{10}^{r}$ found there is compatible with that of Ref. [52, while for $K_{11}^{r}$ the value $2.9 \times 10^{-3}$ is found (with the choices $\mu=\mu_{0}=m_{V}=m_{A} / \sqrt{2}$ ). The latter values, which affect the combination $\left(k_{7}^{r}+2 k_{8}^{r}\right)$, do not seem, however, to qualitatively change the estimate of the correction (6.24) evaluated with the values of the coefficients of Ref. [51]. We shall therefore present the numerical estimates with the values supplied by Ref. [51].

We find (with the mass scale $\mu=m_{\rho}$ ):

$$
\begin{aligned}
& \left(\Delta m_{\pi^{0}}\right)_{\pi \gamma} \simeq-0.01 \mathrm{MeV}, \quad\left(\Delta m_{\pi^{+}}\right)_{\pi \gamma} \simeq 0.43 \mathrm{MeV}, \quad\left(\Delta m_{\pi}\right)_{\pi \gamma}=0.44 \mathrm{MeV} \\
& (\Delta \Gamma)_{\pi \gamma}=-0.20 \alpha \Gamma_{0}=-0.0015 \Gamma_{0} .
\end{aligned}
$$

We observe that the pion mass shift is of the order of $10 \%$ of the observed mass shift $\Delta m_{\pi}=4.6 \mathrm{MeV}$ and has the correct order of magnitude for an $O\left(e^{2} p^{2}\right)$ effect. The decay width correction is found almost equal to zero. The counterterm coefficients $k^{r}$ of Eq. (6.24) give a negative contribution and thus have the tendancy to lower the value of $(\Delta \Gamma)_{\pi \gamma}$; the contribution of the first two terms of the right-hand side of Eq. (6.24) is $0.0025 \Gamma_{0}$. 


\section{$7 \quad$ Electromagnetic mass shift corrections}

This section is devoted to the evaluation of the electromagnetic mass shift corrections contained in the amplitude $\mathcal{R} e \mathcal{M}_{00,+-}^{\text {str. }+q \gamma}$ [Eqs. (4.7)-(4.8)]. We shall proceed in two steps. First, we shall determine the effect of the insertions of the mass term (4.1) in internal charged pion propagators. This will bring us to the strong interaction amplitude calculated with individual momenta fixed at the charged pion mass, but with the mass parameter fixed at the neutral pion mass. In the second step, we shall determine the difference between the latter amplitude and the one calculated with individual momenta and masses fixed at the charged pion mass, with which the numerical values of the scattering lengths are calculated in the literature [15, 223].

For the evaluation of the effect of the insertions of the mass shift term (4.1) the counterterm lagrangians needed here are the quark-photon part of the lagrangian (6.12) and the standard $O\left(p^{4}\right)$ lagrangian [3]; part of the latter survives in the mass shift counterterms. We therefore begin by writing the relevant part of this lagrangian for our problem (with standard notations):

$$
\begin{aligned}
\mathcal{L}^{\left(p^{4}\right)}= & \frac{l_{1}}{4}<\partial_{\mu} U^{\dagger} \partial^{\mu} U>^{2}+\frac{l_{2}}{4}<\partial_{\mu} U^{\dagger} \partial_{\nu} U><\partial^{\mu} U^{\dagger} \partial^{\nu} U> \\
& +\frac{l_{3}}{16}<\chi^{\dagger} U+\chi U^{\dagger}>^{2}+\frac{l_{4}}{4}<\partial_{\mu} \chi^{\dagger} \partial^{\mu} U+\partial_{\mu} \chi \partial^{\mu} U^{\dagger}>.
\end{aligned}
$$

The coefficients $l$ have the following decompositions:

$$
l_{i}=\gamma_{i} \bar{\lambda}+l_{i}^{r}(\mu),
$$

where $\bar{\lambda}$ is defined in Eq. (6.14) and the coefficients $\gamma$ have the values:

$$
\gamma_{1}=\frac{1}{3}, \quad \gamma_{2}=\frac{2}{3}, \quad \gamma_{3}=-\frac{1}{2}, \quad \gamma_{4}=2 .
$$

For the scattering problem the above lagrangian becomes:

$$
\begin{aligned}
\mathcal{L}^{\left(p^{4}\right)=} & \frac{l_{1}}{F_{0}^{4}}\left(\partial_{\mu} \pi^{0} \partial^{\mu} \pi^{0}+2 \partial_{\mu} \pi^{+} \partial^{\mu} \pi^{-}\right)\left(\partial_{\nu} \pi^{0} \partial^{\nu} \pi^{0}+2 \partial_{\nu} \pi^{+} \partial^{\nu} \pi^{-}\right) \\
& +\frac{l_{2}}{F_{0}^{4}}\left(\partial_{\mu} \pi^{0} \partial_{\nu} \pi^{0}+\partial_{\mu} \pi^{+} \partial_{\nu} \pi^{-}+\partial_{\mu} \pi^{-} \partial_{\nu} \pi^{+}\right) \\
& \times\left(\partial^{\mu} \pi^{0} \partial^{\nu} \pi^{0}+\partial^{\mu} \pi^{+} \partial^{\nu} \pi^{-}+\partial^{\mu} \pi^{-} \partial^{\nu} \pi^{+}\right) \\
& -\frac{l_{3}}{F_{0}^{4}}\left(2 \hat{m} B_{0}\right)^{2}\left(\pi^{02}+2 \pi^{+} \pi^{-}\right) .
\end{aligned}
$$


This lagrangian provides the following relationship between the pseudoscalar densities and the pion fields:

$$
P^{a}=\left(2 B_{0} F_{0}\right)\left(1+\frac{2 l_{3}}{F_{0}^{2}} 2 \hat{m} B_{0}\left(1-\frac{\pi^{2}}{2 F_{0}^{2}}\right)-l_{4} \partial^{2}\right) \pi^{a}
$$

The three-pion term does not contribute to the present appreximation to the two-point Green's function renormalization, but provides an additional contribution (a three-propagator term) to the four-point Green's function. Also, the term proportional to $l_{3}$ in Eq. (7.1) provides in addition a two-propagator term to the four-point Green's function. These contributions should be taken into account for the off-mass shell expression of the scattering amplitude.

The finite parts of wave function and mass renormalizations proportional to $C / F_{0}^{4}$ (in notations similar to those of Eqs. (6.18)-(6.19)) are:

$$
\begin{aligned}
-\Delta Z^{0} & =-2 e^{2}\left[k_{2}^{r}-k_{4}^{r}-\frac{20}{9}\left(k_{7}^{r}+k_{11}^{r}\right)\right] \\
\left(\Delta m_{\pi^{0}}^{2}\right)_{q \gamma} & =-2 e^{2} m_{\pi}^{2}\left[-\frac{C}{F_{0}^{4}} \frac{1}{16 \pi^{2}}\left(\ln \left(\frac{m_{\pi}^{2}}{\mu^{2}}\right)+1\right)+k_{2}^{r}-k_{4}^{r}-\frac{10}{9}\left(k_{7}^{r}+k_{11}^{r}\right)\right] \\
-\Delta Z^{+} & =-2 e^{2}\left[\frac{C}{F_{0}^{4}} \frac{1}{16 \pi^{2}}\left(\ln \left(\frac{m_{\pi}^{2}}{\mu^{2}}\right)+1\right)+k_{2}^{r}-\frac{20}{9}\left(k_{7}^{r}+k_{11}^{r}\right)-4 k_{8}^{q \gamma, r}-\frac{2 C}{F_{0}^{4}} l_{4}^{r}\right] \\
\left(\Delta m_{\pi^{+}}^{2}\right)_{q \gamma} & =2 e^{2} \frac{C}{F_{0}^{2}}-2 e^{2} m_{\pi}^{2}\left[\frac{2 C}{F_{0}^{4}} \frac{1}{16 \pi^{2}} \ln \left(\frac{m_{\pi}^{2}}{\mu^{2}}\right)+k_{2}^{r}-\frac{10}{9}\left(k_{7}^{r}+k_{11}^{r}\right)-\left(k_{7}-2 k_{8}^{r}\right)\right] .
\end{aligned}
$$

(We have also incorporated in $\left(\Delta m_{\pi^{+}}^{2}\right)_{q \gamma}$ the lowest order term (4.2)). The difference between the charged and neutral pion masses due to quark-photon interaction is:

$$
\begin{aligned}
\left(\Delta m_{\pi}^{2}\right)_{q \gamma} & =\left(\Delta m_{\pi^{+}}^{2}\right)_{q \gamma}-\left(\Delta m_{\pi^{0}}^{2}\right)_{q \gamma} \\
& =2 e^{2} \frac{C}{F_{0}^{2}}-2 e^{2} m_{\pi}^{2}\left[\frac{C}{F_{0}^{4}} \frac{1}{16 \pi^{4}}\left(3 \ln \left(\frac{m_{\pi}^{2}}{\mu^{2}}\right)+1\right)+k_{4}^{r}-\left(k_{7}^{r}-2 k_{8}^{r}\right)\right] .
\end{aligned}
$$

To evaluate the change in the scattering amplitude due to the pion mass shift (4.2), we first calculate the pion loop contributions with the physical pion masses in the internal propagators. There are diagrams with tadpole loops at the four-pion vertex and four diagrams with loops with two-pion propagators, one with two charged pions, one with two neutral pions and two with one charged and one neutral pions. To the contributions of these diagrams one adds the remaining contributions of the pseudoscalar densities to the 
four-point vertex function [Eqs. (6.17) and (7.5)]. The divergences of the above contributions are cancelled by those of the counterterm lagrangians (6.16) (the part proportional to the factor $C / F_{0}^{4}$ ) and (7.4). One multiplies the result by the wave function renormalization factors $\left(1-\Delta Z^{0} / 2\right)^{2}$ and $\left(1-\Delta Z^{+} / 2\right)^{2}$ coming from the contributions (7.6)-(7.7) and from those of the strong interaction limit [3] and isolates in the resulting expression the part proportional to $C / F_{0}^{4}$. One also incorporates in the mass term of the lowest-order amplitude (1.1) the neutral pion mass shift (7.6). One finds in the limits $t=u=0$ (with the notations $m_{\pi}=m_{\pi^{0}}$ and $\Delta m_{\pi}^{2}=\left(\Delta m_{\pi}^{2}\right)_{q \gamma}$, Eq. (7.8)):

$$
\begin{aligned}
F_{0}^{4}( & \left.\Delta \mathcal{R}_{e} \mathcal{M}_{00,+-}\right)_{q \gamma}= \\
& -\frac{1}{32 \pi^{2}}\left(s-m_{\pi}^{2}\right)\left(s+p_{1}^{2}+p_{2}^{2}-2 m_{\pi}^{2}\right)\left(\frac{\Delta m_{\pi}^{2}}{m_{\pi}^{2}}+\mathcal{R} e\left(Q^{+-}(s)-Q^{00}(s)\right)\right) \\
& +\frac{1}{16 \pi^{2}}\left(s-m_{\pi}^{2}\right)\left(2-\mathcal{R} e Q^{00}(s)\right) \Delta m_{\pi}^{2}-\frac{1}{64 \pi^{2}}\left(p_{1}^{2}+p_{2}^{2}\right)\left(p_{3}^{2}+p_{4}^{2}\right) \frac{\Delta m_{\pi}^{2}}{m_{\pi}^{2}} \\
& +2 e^{2} F_{0}^{2}\left(3 k_{2}^{r}-2 k_{4}^{r}\right) s-2 e^{2} F_{0}^{2}\left(2 k_{2}^{r}-k_{4}^{r}\right)\left(p_{3}^{2}+p_{4}^{2}\right) \\
& -2 e^{2} F_{0}^{2}\left(k_{2}^{r}-k_{4}^{r}-\left(k_{7}^{r}-2 k_{8}^{r}\right)\right) m_{\pi}^{2}-2 e^{2} F_{0}^{2}\left(2 k_{2}^{r}-k_{4}^{r}\right)\left(s-m_{\pi}^{2}\right) \\
& +2 e^{2} F_{0}^{2}\left(k_{7}^{r}-2 k_{8}^{r}\right)\left(p_{3}^{2}+p_{4}^{2}-2 m_{\pi}^{2}\right) \\
& -4 l_{3}^{r}\left(p_{3}^{2}+p_{4}^{2}-m_{\pi}^{2}\right) \Delta m_{\pi}^{2}-2 l_{4}^{r}\left(s-m_{\pi}^{2}\right) \Delta m_{\pi}^{2} .
\end{aligned}
$$

(Details of the calculations can be found in Appendix E. $Q(s)$ is defined in Eq. (E.3). Only relevant first-order terms in $\Delta m_{\pi}^{2}$ have been kept.)

With the mass shell conditions $p_{i}^{2}=m_{\pi^{+}}^{2}(i=1, \ldots, 4)$ and $s=4 m_{\pi^{+}}^{2}$, the above expression becomes, to first-order in $\Delta m_{\pi}^{2}$ :

$$
\begin{aligned}
F_{0}^{4}\left(\Delta \mathcal{R} e \mathcal{M}_{00,+-}\left(s=4 m_{\pi^{+}}^{2}\right)\right)_{q \gamma}= & \frac{11 m_{\pi}^{2}}{16 \pi^{2}} \Delta m_{\pi}^{2}-\left(4 l_{3}^{r}+6 l_{4}^{r}\right) m_{\pi}^{2} \Delta m_{\pi}^{2} \\
& +2 e^{2} F_{0}^{2}\left(\left(k_{2}^{r}-2 k_{4}^{r}\right)+\left(k_{7}^{r}-2 k_{8}^{r}\right)\right) m_{\pi}^{2}
\end{aligned}
$$

The relationships between the $k_{i}^{r}$ 's and the $S U(3)$ coefficients $K_{i}^{r}$ [45] are $\left(m_{K}\right.$ is the kaon mass):

$$
\begin{aligned}
k_{2}^{r} & =K_{2}^{r}+K_{6}^{r}-\frac{1}{64 \pi^{2}}\left(\ln \left(\frac{m_{K}^{2}}{\mu^{2}}\right)+1\right), \quad k_{4}^{r}=-K_{4}^{r}, \\
k_{7}^{r}+k_{11}^{r} & =\frac{1}{5}\left(6 K_{8}^{r}+5 K_{9}^{r}+5 K_{10}^{r}\right)-\frac{9}{320 \pi^{2}}\left(\ln \left(\frac{m_{K}^{2}}{\mu^{2}}\right)+1\right), \\
k_{7}^{r}-2 k_{8}^{r} & =10 K_{8}^{r}-\frac{3}{16 \pi^{2}}\left(\ln \left(\frac{m_{K}^{2}}{\mu^{2}}\right)+1\right) .
\end{aligned}
$$


Numerical values for the coefficients $K_{i}^{r}$ are presented in Ref. [51]. They are in units of $10^{-3}$ and at the scale $\mu=m_{\rho}: K_{2}^{r}=-3.1, K_{4}^{r}=-6.2, K_{6}^{r}=8.6, K_{8}^{r}=K_{9}^{r}=K_{10}^{r}=0$. The values of the coefficients $l_{i}^{r}$ can be found in Ref. [15]. They are in units of $10^{-3}$ and at the scale $\mu=m_{\rho}: l_{1}^{r}=-5.4, l_{2}^{r}=5.67, l_{3}^{r}=0.82, l_{4}^{r}=5.6$. One finds the following corrections in the scattering lengths and the decay width:

$$
\left(\Delta\left(a_{0}^{0}-a_{0}^{2}\right)\right)_{q \gamma}=0.0005, \quad \frac{(\Delta \Gamma)_{q \gamma}}{\Gamma_{0}}=0.0035 .
$$

Once the correction due to the mass shift (4.2) is isolated, the amplitude $\mathcal{R} e \mathcal{M}_{00,+-}^{\text {str. }+q \gamma}$ reduces to the strong interaction amplitude calculated with individual momenta fixed at the charged pion mass [Eq. (4.8)] and the mass parameter fixed at the neutral pion mass. Numerical values of the strong interaction scattering lengths are calculated in the literature with the individual momenta and the mass parameter fixed at the charged pion mass [15, 23]. It is therefore necessary to evaluate the difference between these two amplitudes. It can be obtained from the $O\left(p^{4}\right)$ off-mass shell expression of the scattering amplitude given in Ref. [3] (we neglect for the moment the mass shift effect in the $O\left(p^{6}\right)$ term). Replacing in the $O\left(p^{2}\right)$ term (1.1) the mass parameter in terms of $m_{\pi^{0}}^{2}$ with the use of the relation [3]:

$$
m_{\pi^{0}}^{2}=2 \hat{m} B_{0}+\frac{m_{\pi}^{4}}{F_{0}^{2}}\left(2 l_{3}^{r}+\frac{1}{32 \pi^{2}} \ln \left(\frac{m_{\pi}^{2}}{\mu^{2}}\right)\right),
$$

one finds for the shift at threshold from the amplitude calculated with the charged pion mass:

$$
\begin{gathered}
\left(\Delta \mathcal{R} e \mathcal{M}_{00,+-}\left(s=4 m_{\pi^{+}}^{2}\right)\right)_{\Delta m_{\pi}}=\frac{\Delta m_{\pi}^{2}}{F_{0}^{2}}\left[1+\frac{m_{\pi}^{2}}{F_{0}^{2}}\left(-\frac{9}{32 \pi^{2}}-\frac{11}{16 \pi^{2}} \ln \left(\frac{m_{\pi}^{2}}{\mu^{2}}\right)\right.\right. \\
\left.\left.+4 l_{3}^{r}+12 l_{4}^{r}\right)\right] .
\end{gathered}
$$

Designating by $\mathcal{M}_{00,+-}^{\text {str. }}$ the strong interaction amplitude (without mass shifts), one has at threshold [Eqs. (3.5), (3.6) and (3.7)]:

$$
\mathcal{M}_{00,+-}^{\text {str. }}\left(s=4 m_{\pi}^{2}\right)=\frac{32 \pi}{3}\left(a_{0}^{0}-a_{0}^{2}\right)_{s t r .}
$$

where the $a$ 's are the scattering lengths calculated up to two loops [15, 23] of the chiral effective lagrangian. In the standard scheme one has the value [3, 15] : $\left(a_{0}^{0}-a_{0}^{2}\right)=0.258$, obtained with charged pion masses. Eq. (7.14) yields the following corrections for the 
scattering lengths and the decay width (we take $F_{0}=88 \mathrm{MeV}, m_{\pi^{+}}=139.57 \mathrm{MeV}$, $\left.\Delta m_{\pi}=4.6 \mathrm{MeV}\right)$ :

$$
\left(\Delta\left(a_{0}^{0}-a_{0}^{2}\right)\right)_{\Delta m_{\pi}}=0.0083, \quad \frac{(\Delta \Gamma)_{\Delta m_{\pi}}}{\Gamma_{0}}=0.064 .
$$

The contribution of the $O\left(p^{4}\right)$ term represents $40 \%$ of this correction, indicating an increase of its relative strength by a factor of 2 with respect to the corresponding situation in the amplitude; this might be understood as a consequence of the increase of the powers of mass and momemtum terms in higher-order terms. In this case one should expect a correction of the order of $3 \times 5 \%$ coming from the $O\left(p^{6}\right)$ term, which would bring the correction (7.16) in the scattering lengths to 0.0095 .

The effective scattering length corresponding to the amplitude $\mathcal{R} e \mathcal{M}_{00,+-}^{\text {str.+q }}$ (4.7) is then (without the use of the $O\left(e^{2} p^{4}\right)$ correction coming from the $O\left(p^{6}\right)$ term):

$$
\left(a_{0}^{0}-a_{0}^{2}\right)_{s t r .+q \gamma}=\left(a_{0}^{0}-a_{0}^{2}\right)_{s t r .\left(\pi^{+}\right)}+\left(\Delta\left(a_{0}^{0}-a_{0}^{2}\right)\right)_{q \gamma}+\left(\Delta\left(a_{0}^{0}-a_{0}^{2}\right)\right)_{\Delta m_{\pi}}=0.267 .
$$

(The inclusion of the estimated $O\left(e^{2} p^{4}\right)$ correction coming from the $O\left(p^{6}\right)$ term would bring this number to 0.268.)

It is also possible to extract the value of the constant $C$ from the pion mass shift formula (7.8). Taking into account the mass shifts due to pion-photon interaction (0.4 $\mathrm{MeV}$, Eqs. (6.20) and (6.26) ) and to isospin breaking (0.2 MeV [3, (4) and the observed pion mass difference $(4.6 \mathrm{MeV})$, one finds $\left(\Delta m_{\pi}\right)_{q \gamma} \simeq 4.0 \mathrm{MeV}$, leading to:

$$
C=4.1 \times 10^{-5} \mathrm{GeV}^{4}
$$

This value is in agreement with the central value found in Ref. [52] at a slightly higher mass scale $\left((4.2 \pm 1.5) \times 10^{-5} \mathrm{GeV}^{4}\right.$, at $\left.\mu=0.85 \mathrm{GeV}\right)$. The corresponding $O\left(e^{2} p^{0}\right)$ mass shift is $\left(\Delta m_{\pi}\right)_{q \gamma}^{\left(e^{2} p^{0}\right)} \simeq 3.6 \mathrm{MeV}$, the remaining $0.4 \mathrm{MeV}$ being produced by the $O\left(e^{2} p^{2}\right)$ correction [Eq. (7.8)], with $\left(\Delta m_{\pi^{0}}\right)_{q \gamma}=-0.13 \mathrm{MeV}$.

\section{Summary}

The total amount of $O(\alpha)$ corrections to the lowest-order formula of the pionium decay width can be represented in the following form:

$$
\Gamma=\Gamma_{0} \sqrt{\left(1-\frac{\Delta m_{\pi}}{2 m_{\pi^{+}}}\right)}\left(1+\frac{(\Delta \Gamma)_{s t r .}}{\Gamma_{0}}\right)\left(1+\frac{(\Delta \Gamma)_{\pi \gamma}}{\Gamma_{0}}\right)\left(1+\frac{(\Delta \Gamma)_{q \gamma}}{\Gamma_{0}}\right)\left(1+\frac{(\Delta \Gamma)_{\Delta m_{\pi}}}{\Gamma_{0}}\right),
$$


where $\Gamma_{0}$ is the lowest-order decay width, Eq. (1.2), with the strong interaction scattering lengths calculated up to two-loop order of the chiral effective lagrangian with charged pion masses; $(\Delta \Gamma)_{s t r}$. is the correction arising from the second-order perturbation theory contribution of the interaction potential; $(\Delta \Gamma)_{\pi \gamma}$ arises from the radiative corrections due to pion-photon interaction; $(\Delta \Gamma)_{q \gamma}$ is the correction coming from the pion mass shift due to quark-photon interaction; $(\Delta \Gamma)_{\Delta m_{\pi}}$ is the correction coming from the shift in the strong interaction amplitude due to the passage from the charged pion masses to the neutral pion masses with individual momenta fixed at the charged pion mass. The corresponding numerical values are (in the standard scheme):

$$
\begin{array}{rlrl}
\frac{(\Delta \Gamma)_{s t r .}}{\Gamma_{0}} & \simeq 0.004, & & \frac{(\Delta \Gamma)_{\pi \gamma}}{\Gamma_{0}} \simeq-0.001 \\
\frac{(\Delta \Gamma)_{q \gamma}}{\Gamma_{0}} & \simeq 0.003, & \frac{(\Delta \Gamma)_{\Delta m_{\pi}}}{\Gamma_{0}} \simeq 0.064
\end{array}
$$

These numbers are subject to uncertainties coming from the numerical values of the coefficints $k_{i}^{r}$ and $l_{i}^{r}$ of the counterterm lagrangians and from the higher loop effects not considered in the pion-photon and quark-photon interactions, corresponding to $O\left(e^{2} p^{4}\right)$ terms. These corrections should not qualitatively change the above numerical estimates. One finds for the lifetime the value:

$$
\tau=2.97 \times 10^{-15} \mathrm{~s}
$$

to be compared with the value $\tau_{0}=3.19 \times 10^{-15} \mathrm{~s}$ found from formula (1.2) using $\left(a_{0}^{0}-\right.$ $\left.a_{0}^{2}\right)=0.258$.

The evaluation of the corrections in the generalized scheme is more complicated because of the presence of additional terms in the counterterm lagrangians. However, their order of magnitude should not qualitatively differ from those of the standard scheme.

\section{Acknowledgements}

We are grateful to L. L. Nemenov for useful informations on the DIRAC project. We thank M. Knecht, B. Moussallam and J. Stern for stimulating discussions. One of us (H.S.) thanks J. Gasser and H. Leutwyler for several helpful and clarifying discussions. He also enjoyed discussions with E. A. Kuraev, P. Minkowski and A. G. Rusetsky. 


\section{A Integrals of the strong interaction constraint diagrams}

This appendix is devoted to the calculation of the constraint diagrams entering in the strong interaction corrections to the potentials (Sec. 4 and Fig. 1).

The incoming particles are designated by 1 and 2, the outgoing ones by 3 and 4, the loop particles by 5 and 6 . They represent either $\pi^{+} \pi^{-}$or $\pi^{0} \pi^{0}$. The relative momentum of the ingoing particles is designated by $p$, that of the outgoing ones by $p^{\prime}$. The loop particles have momenta $p_{1}-k$ and $p_{2}+k$. The constraint (2.1) implies that $p_{i L}=P_{L} / 2$ $(i=1, \ldots, 6)$ and $k_{L}=0$ inside the loop.

The various momentum transfers have the following values:

$$
\begin{aligned}
& t_{51}=\left(p_{1}-p_{5}\right)^{2}=k^{T 2}, \quad u_{61}=\left(p_{1}-p_{6}\right)^{2}=\left(2 p^{T}-k^{T}\right)^{2}, \\
& t_{35}=\left(p_{5}-p_{3}\right)^{2}=\left(p^{\prime T}-p^{T}+k^{T}\right)^{2}, \quad u_{45}=\left(p_{5}-p_{4}\right)^{2}=\left(p^{\prime T}+p^{T}-k^{T}\right)^{2} .
\end{aligned}
$$

We also use notations (2.9) with $b^{2}=-p^{T 2}$ and $b^{\prime 2}=-p^{\prime T 2}$. We choose for the evaluation of the integrals values of $s$ below $4 m_{\pi}^{2}, m_{\pi}$ designating here the mass of the loop pion, so that $b_{0}^{2}$ is negative. The analytic continuation of $\sqrt{-b_{0}^{2}}$ to positive values of $b_{0}^{2}$ is done with

the replacement $\sqrt{-b_{0}^{2}} \rightarrow-i \sqrt{b_{0}^{2}}$. We use for the integrations dimensional regularization.

We introduce the compact notation:

$$
\left(a \widetilde{g}_{0} b\right)=\int \frac{d^{3} k^{T}}{(2 \pi)^{3}} a \widetilde{g}_{0}\left(\frac{\sqrt{s}}{2}, p^{T}-k^{T}\right) b
$$

where $a$ and $b$ represent either one of the momenta $t$ and/or $u$ defined above or the factor 1. We find:

$$
\begin{aligned}
\left(\widetilde{g}_{0}\right) & =-\int \frac{d^{3} k^{T}}{(2 \pi)^{3}} \frac{1}{b_{0}^{2}+\left(p^{T}-k^{T}\right)^{2}+i \epsilon}=-\frac{1}{4 \pi} \sqrt{-b_{0}^{2}} \\
\left(\widetilde{g}_{0} t_{51}\right) & =\left(\widetilde{g}_{0} u_{61}\right)=\frac{1}{4 \pi}\left(b^{2}+b_{0}^{2}\right) \sqrt{-b_{9}^{2}} \\
\left(t_{35} \widetilde{g}_{0}\right) & =\left(u_{45} \widetilde{g}_{0}\right)=\frac{1}{4 \pi}\left(b^{\prime 2}+b_{0}^{2}\right) \sqrt{-b_{0}^{2}} \\
\left(t_{35} \widetilde{g}_{0} t_{51}\right) & =\left(u_{45} \widetilde{g}_{0} u_{61}\right)=-\frac{1}{4 \pi}\left[\frac{2}{3} t b_{0}^{2}+b^{2} b^{\prime 2}+\frac{5}{3} b_{0}^{2}\left(b^{2}+b^{2}\right)+\left(b_{0}^{2}\right)^{2}\right] \sqrt{-b_{0}^{2}} \\
\left(t_{35} \widetilde{g}_{0} u_{61}\right) & =\left(u_{45} \widetilde{g}_{0} t_{51}\right)=-\frac{1}{4 \pi}\left[\frac{2}{3} u b_{0}^{2}+b^{2} b^{\prime 2}+\frac{5}{3} b_{0}^{2}\left(b^{2}+b^{\prime 2}\right)+\left(b_{0}^{2}\right)^{2}\right] \sqrt{-b_{0}^{2}}
\end{aligned}
$$


Equations (A.4) show that the integrals involving the variables $t$ and/or $u$ yield, with $\widetilde{g}_{0,+-}$, quantities that are of order $O\left(\alpha^{3}\right)$ and higher and thus are negligible. The similar terms considered with $\widetilde{g}_{0,00}$ yield contributions of order $O\left(\alpha^{2} \sqrt{\frac{\Delta m_{\pi}}{m_{\pi}}}\right)$, or $O\left(\left(\frac{\Delta m_{\pi}}{m_{\pi}}\right)^{3 / 2}\right)$ and higher, which also are imaginary. Apart from being small, these terms will cancel the imaginary part of the amplitude present in the expression of the potential. The leading $O(\alpha)$ or $O\left(\sqrt{\frac{\Delta m_{\pi}}{m_{\pi}}}\right)$ terms of the constraint diagrams are simply obtained by neglecting from the start the variables $t$ and $u$ in the scattering amplitudes, which then factorize outside the integrals and the latter reduce to the integral over the constraint propagator $\tilde{g}_{0}[\mathrm{Eq} \cdot(\mathrm{A} .3)]$.

\section{B Two-loop diagrams}

In this appendix we generalize the validity of the lowest-order formula established in Sec. 4 [Eq. (4.7)] to the two-loop level.

The main ingredient of the proof is already met at the one-loop level and can be summarized as follows. In the vicinity of the two-pion threshold, the strong interaction unitarity one-loop amplitude (with the pion mass shift included in) can be decomposed into an analytic function $\mathcal{M}_{a n}^{(1)}$. of the real variables $b_{0}^{2}(s), t$ and $u$ and a non-analytic part, $\mathcal{M}_{\text {nan. }}^{(1)}$ (essentially proportional to $\sqrt{-b_{0}^{2}}$ ). [The analyticity of $\mathcal{M}_{a n \text {. }}^{(1)}$ in $t$ and $u$ near threshold is due to the absence of infra-red singularities in the strong interaction amplitude with massive pions.] The constraint diagram amplitude, $\mathcal{M}^{C(1,0,1)}$ (the notation being explained before Eq. (5.8)), has the property of cancelling the nonanalytic part, $\mathcal{M}_{\text {nan }}^{(1)}$, of the one-loop amplitude (sum of the two diagrams of Fig. 1):

$$
\mathcal{M}^{(1)}+\mathcal{M}^{C(1,0,1)}=\mathcal{M}_{a n .}^{(1)}+\mathcal{M}_{\text {nan. }}^{(1)}+\mathcal{M}^{C(1,0,1)}=\mathcal{M}_{a n .}^{(1)}
$$

The deviation of the analytic piece, $\mathcal{M}_{\text {an. }}^{(1)}$, from the pionium energy to the $\pi^{+} \pi^{-}$threshold is of order $O\left(\alpha^{2}\right)$ and hence $\mathcal{M}_{a n}^{(1)}$. can be replaced by its value at threshold:

$$
\mathcal{M}_{a n .}^{(1)}\left(b_{0}^{2}, t, u\right)=\mathcal{M}_{a n .}^{(1)}\left(s=4 m_{\pi^{+}}^{2}, t=0, u=0\right)+O\left(\alpha^{2}\right) .
$$

Two cases must be distinguished, depending on whether the loop is made of $\pi^{+} \pi^{-}$or of $\pi^{0} \pi^{0}$. In the first case $\left(\pi^{+} \pi^{-}\right.$-loop $)$, the nonanalytic piece, $\mathcal{M}_{\text {nan. }}^{(1)}$, and the constraint amplitude, $\mathcal{M}^{C(1,0,1)}$, are real and separately vanish at the $\pi^{+} \pi^{-}$threshold and therefore 
the value of $\mathcal{M}_{a n}^{(1)}$ at threshold coincides with that of the one-loop amplitude $\mathcal{M}^{(1)}$ at threshold, which is real. In the second case $\left(\pi^{0} \pi^{0}\right.$-loop $), \mathcal{M}_{\text {nan. }}^{(1)}$ and $\mathcal{M}^{C(1,0,1)}$ are imaginary and do not vanish at the $\pi^{+} \pi^{-}$threshold; the value of $\mathcal{M}_{a n}^{(1)}$. then coincides with the real part of the one-loop amplitude $\mathcal{M}^{(1)}$ at threshold. Therefore, in all cases we have:

$$
\mathcal{M}^{(1)}+\mathcal{M}^{C(1,0,1)}=\mathcal{R} e \mathcal{M}^{(1)}\left(s=4 m_{\pi^{+}}^{2}, t=0, u=0\right) .
$$

This property can be used for the analysis of two-loop diagrams. It is clear that not all two-loop diagrams necessitate a detailed study; those not having singularities in the $s$-channel are analytic in the vicinity of the two-pion threshold and real; since in this case their deviation from the threshold value is of order $O\left(\alpha^{2}\right)$, they can immediately be replaced by their value at the $\pi^{+} \pi^{-}$threshold.

The typical diagrams involved in this analysis are presented in Fig. 4.

The sum of the four diagrams of Fig. 4a (where the loop pions are the same in the four diagrams) is free of singularities in the $s$-channel and is represented in the vicinity of the two-pion threshold by a real analytic function. The value of the latter at the pionium energy differs from its value at the $\pi^{+} \pi^{-}$threshold by an $O\left(\alpha^{2}\right)$ term; hence it can be replaced by its value at the two-pion threshold. When the loops contain at least one pair of $\pi^{+} \pi^{-}$, this value coincides with the real part of the two-loop amplitude (first diagram) at threshold. If the two loops correspond to neutral pions, then this value differs from the real part of the amplitude at threshold by a factor that is proportional to the product of the imaginary parts of each loop (actually cancelled by the last constraint diagram) and hence to $\left(\Delta m_{\pi}\right)^{2}$. This factor contributes with a relative order of magnitude of $10^{-4}$ and can be neglected. Therefore, the sum of the four diagrams at the pionium energy is equivalent to the real part of the two-loop amplitude (first diagram) at the $\pi^{+} \pi^{-}$ threshold.

In diagrams of Fig. 4b, the tadpole diagram factorizes at the vertex and does not interfere with the loop diagram calculations. For the latter, one has the same results as in the one-loop case (Fig. 1).

In the first diagram of Fig. 4c, the internal propagator is modified with the inclusion of the self-energy correction (which must also be done in the other propagator). This feature does not qualitatively change the singularities of the diagram with respect to the one-loop case, for these depend essentially on the mass-shell condition of the internal loops. A similar self-energy inclusion in the constraint propagator [Eq. (2.7)] (second diagram) 
ensures the cancellation of the singularities of the first diagram and the reasonings of the one-loop case can be repeated. (The mass shift coming from the above self-energy correction can be incorporated in the mass term used in the constraint propagator $\widetilde{g}_{0}$. Wave function renormalization constants should not influence physical results.)

In the diagrams of Fig. 4d, the constraint diagram, corresponding to the first loop, cancels the singularities of the first loop of the first diagram. The second loop, which is on the right of each diagram, is free of singularities in the $s$-channel and is also free of infra-red singularities (no massless particles). Hence, the sum of the two diagrams is analytic in the vicinity of the two-pion threshold and the previous results are found again.

There are also diagrams with three internal pion propagators, in which three external pions join each other at one vertex and a single external pion is attached at the other vertex. Such diagrams have singularities for $p_{i}^{2} \geq\left(3 m_{\pi}\right)^{2}(i=1, \ldots, 4)$. For $p_{i}^{2}$ 's in the vicinity of the mass shell, as is the case in the present problem, these diagrams are free of singularities and are analytic in $s, t, u$.

The remaining two-loop diagrams do not have singularities in the $s$-channel and the above diagrams exhaust the cases where constraint diagrams occur. The result found in Sec. 4 [Eq. (4.7)] can therefore be generalized to the two-loop level.

At the three-loop level, a qualitative change appears with the occurrence of inelasticities through the four-pion intermediate states. The constraint propagator (2.7) is no longer sufficient by itself to cancel the singularities of the scattering amplitude. In this case, new pieces should be added to it to take into account the inelasticity effects.

\section{Cancellation of linear divergences}

When the linear divergence of the continuous spectrum is kept, $(\Delta \Gamma)_{\text {cont. }}$ [Eq. (5.5)] receives the contribution:

$$
(\Delta \Gamma)_{\text {cont. }}=-2 \Gamma_{0} \bar{V}_{+-,+-} \int_{0}^{\infty} \frac{d k}{2 \pi^{2}}+\cdots,
$$

where the dots stand for the contributions other than that of the linear divergence.

On the other hand, the integration of the constraint propagator $\widetilde{g}_{0}$ [Eq. (2.7)] also produces a linear divergence. Equation (A.3) now becomes:

$$
\left(\widetilde{g}_{0}\right)=-\int \frac{d^{3} k^{T}}{(2 \pi)^{3}} \frac{1}{b_{0}^{2}+\left(p^{T}-k^{T}\right)^{2}+i \epsilon}=\int_{0}^{\infty} \frac{d k}{2 \pi^{2}}-\frac{1}{4 \pi} \sqrt{-b_{0}^{2}} .
$$


The corresponding linear divergence shows up in the contributions of constraint diagrams that enter in the calculation of the potentials. We first consider the constraint diagram $\bar{V}_{+-,+-} \widetilde{g}_{0 .+-} \widetilde{T}_{+-, 00}$ (in lowest order) contributing to $V_{+-, 00}$ in Eq. (3.8). The linear divergence coming from $\widetilde{g}_{0,+-}$ [Eq. (C.2)] gives an additional contribution to $V_{+-, 00}$ :

$$
V_{+-, 00}=\bar{V}_{+-,+-} V_{+-, 00} \int_{0}^{\infty} \frac{d k}{2 \pi^{2}}+\cdots .
$$

This quantity, when inserted in the last term of the wave equation (3.13), provides, in first-order of perturbation theory, the contribution to the decay width:

$$
(\Delta \Gamma)^{C(2,0,2)}=2 \Gamma_{0} \bar{V}_{+-,+-} \int_{0}^{\infty} \frac{d k}{2 \pi^{2}},
$$

which cancels the linearly divergent contribution (C.1). In the last term of Eq. (3.13) there are also quadratic terms in $\int d k /\left(2 \pi^{2}\right)$, which however are proportional to $\bar{V}_{+-,+-}^{2}$. These must be associated with similar divergences arising from third-order perturbation theory. The constraint diagrams of Fig. 2 considered in Sec. 5 do not contain linear divergences and hence the net result of Eq. (5.15) remains unchanged.

The other linear divergences present in the constraint diagram contributions must similarly be associated with other contributions having the same types of divergences. Thus, the linear divergence of the term $\bar{V}_{+-,+-} \widetilde{g}_{0,+-} \bar{V}_{+-,+-}$which enters in the calculation of $V_{+-,+-}[$Eq. (3.8)] must be associated with the second-order perturbation theory contribution of $\bar{V}_{+-,+-}$of the wave equation (3.13) which concerns the shift in the real part of the energy. The linear divergence of the term $\frac{1}{2} \bar{V}_{+-, 00} \widetilde{g}_{0,00} V_{00,+-}$, contributing to $V_{+-,+-}$ cancels a similar divergence coming from the last term of the wave equation (3.13). The linear divergence of the term $\frac{1}{2} V_{+-, 00} \widetilde{g}_{0,00} V_{00,00}$ contributing to the calculation of $V_{+-, 00}$ must be associated with the contribution of the last term of Eq. (3.11) (and which was neglected in the text because of the smallness of its finite part; cf. the paragraph preceding Eq. (3.13)).

In summary, the contributions of linear divergences, when isolated and grouped in terms with definite structeres, disappear by mutual cancellations from physical quantities. In this respect, the use of dimensional regularization automatically takes into account from the start the above cancellations. 


\section{Integrals of the radiative corrections}

We present in this appendix the definitions and expressions of the integrals involved in the electromagnetic radiative corrections. For the evaluation of the divergent integrals we use dimensional regularization and designate by $d$ and $\mu$ the space-time dimension and the regularization mass, respectively.

The integrals involved in the self-energy part are the following:

$$
\begin{aligned}
T(p) & =\mu^{4-d} \int \frac{d^{d} k}{(2 \pi)^{d}} \frac{1}{(p-k)^{2}-m_{\pi}^{2}+i \epsilon}=i m_{\pi}^{2}\left(\frac{1}{\varepsilon}+\frac{1}{16 \pi^{2}}\right), \\
R_{, \mu, \mu \nu}^{(n)}(p) & =\mu^{4-d} \int \frac{d^{d} k}{(2 \pi)^{d}} \frac{\left(1, k_{\mu}, k_{\mu} k_{\nu}\right)}{(p-k)^{2}-m_{\pi}^{2}+i \epsilon} \frac{1}{\left(k^{2}+i \epsilon\right)^{n}}, \\
R^{(1)}(p) & =\frac{i}{\varepsilon}+\frac{i}{16 \pi^{2}}\left[2-\frac{\lambda^{2}}{p^{2}} \ln \left(\frac{m_{\pi}^{2}}{\lambda^{2}}\right)\right], \\
R_{\mu}^{(1)}(p) & =\frac{i}{2 \bar{\epsilon}} p_{\mu}+\frac{i}{32 \pi^{2}} p_{\mu}\left[1-\frac{\lambda^{2}}{p^{2}}+\left(\frac{\lambda^{2}}{p^{2}}\right)^{2} \ln \left(\frac{m_{\pi}^{2}}{\lambda^{2}}\right)\right], \\
R_{\mu \nu}^{(2)}(p) & =\frac{i}{4 \bar{\epsilon}} g_{\mu \nu}+\frac{i}{64 \pi^{2}} g_{\mu \nu}\left(3+\frac{\lambda^{2}}{p^{2}}-\frac{2 \lambda^{2}}{p^{2}} \ln \left(\frac{m_{\pi}^{2}}{\lambda^{2}}\right)-\left(\frac{\lambda^{2}}{p^{2}}\right)^{2} \ln \left(\frac{m_{\pi}^{2}}{\lambda^{2}}\right)\right) \\
& \quad-\frac{i}{32 \pi^{2}} \frac{p_{\mu} p_{\nu}}{p^{2}}\left(\frac{\lambda^{2}+m_{\pi}^{2}}{p^{2}}-2 \frac{\lambda^{2} m_{\pi}^{2}}{\left(p^{2}\right)^{2}} \ln \left(\frac{m_{\pi}^{2}}{\lambda^{2}}\right)\right),
\end{aligned}
$$

where we have defined:

$$
\begin{aligned}
\frac{1}{\varepsilon} & =\frac{1}{16 \pi^{2}}\left[\frac{1}{2-d / 2}-\ln \left(\frac{m_{\pi}^{2}}{\mu^{2}}\right)+\Gamma^{\prime}(1)+\ln (4 \pi)\right], \\
\lambda^{2} & =m_{\pi^{2}}-p^{2} .
\end{aligned}
$$

One finds for the self-energy the expression:

$$
\begin{aligned}
\Sigma(p)=\frac{e^{2}}{\varepsilon}\left(3 m_{\pi}^{2}\right. & \left.-(2+\xi) \lambda^{2}\right)+\frac{\alpha}{\pi}\left[\frac{7}{4} m_{\pi}^{2}-\lambda^{2}\right. \\
& \left.-\left(1+\frac{\xi}{2}\right) \lambda^{2} \ln \left(\frac{m_{\pi}^{2}}{\lambda^{2}}\right)-\frac{1}{2}\left(1+\frac{\xi}{2}\right) \frac{\left(\lambda^{2}\right)^{2}}{p^{2}} \ln \left(\frac{m_{\pi}^{2}}{\lambda^{2}}\right)\right] .
\end{aligned}
$$

The integrals involved in the vertex function are the following:

$$
\begin{aligned}
F_{, \mu}^{(n)}\left(p_{1}, p_{2}, q\right) & =\mu^{4-d} \int \frac{d^{d} k}{(2 \pi)^{d}} \frac{\left(1, k_{\mu}\right)}{\left(\left(p_{1}-k\right)^{2}-m_{\pi}^{2}+i \epsilon\right)\left(\left(p_{2}+k\right)^{2}-m_{\pi}^{2}+i \epsilon\right)\left(k^{2}+i \epsilon\right)^{n}}, \\
\bar{R}\left(p_{1}, p_{2}\right) & =\mu^{4-d} \int \frac{d^{d} k}{(2 \pi)^{d}} \frac{1}{\left(\left(p_{1}-k\right)^{2}-m_{\pi}^{2}+i \epsilon\right)\left(\left(p_{2}+k\right)^{2}-m_{\pi}^{2}+i \epsilon\right)} .
\end{aligned}
$$


Their expressions are:

$$
\begin{aligned}
& F^{(1)}\left(p_{1}, p_{2}, 0\right)=-\frac{i}{8 \pi s} \sqrt{\frac{s}{b^{2}}} \arctan \sqrt{\frac{b^{2}}{-b_{0}^{2}}} \\
& +\frac{i}{8 \pi^{2} s}\left(2 \ln \left(\frac{s}{\lambda^{2}}\right)-4 \ln 2+4\right), \quad s \simeq 4 m_{\pi}^{2}, \\
& F^{(1)}\left(p_{1},-p_{1}, 0\right)=-\frac{i}{16 \pi^{2}} \frac{1}{p_{1}^{2}} \ln \left(\frac{m_{\pi}^{2}}{\lambda^{2}}\right) \\
& F_{\mu}^{1)}\left(p_{1}, p_{2}, 0\right)=-\frac{i}{16 \pi^{2} s} p_{\mu}^{T}\left[-4+\pi \sqrt{\frac{s}{b^{2}}}\left(\frac{\lambda^{2}}{b^{2}} \arctan \sqrt{\frac{b^{2}}{-b_{0}^{2}}}-\sqrt{\frac{-b_{0}^{2}}{b^{2}}}\right)\right], \quad s \simeq 4 m_{\pi}^{2}, \\
& F_{\mu}^{(1)}\left(p_{1},-p_{1}, 0\right)=-\frac{i}{32 \pi^{2}} p_{1 \mu}\left[\frac{1}{m_{\pi}^{2}}+\frac{1}{p_{1}^{2}}-2 \frac{\lambda^{2}}{\left(p_{1}^{2}\right)^{2}} \ln \left(\frac{m_{\pi}^{2}}{\lambda^{2}}\right)+\frac{\lambda^{2}}{m_{\pi}^{2} p_{1}^{2}}\right], \\
& F_{\mu}^{(2)}\left(p_{1}, p_{2}, 0\right)=\frac{i}{32 \pi^{2}}\left(p_{1}-p_{2}\right)_{\mu} \frac{\pi}{s^{2}}\left(\left(\frac{s}{b^{2}}\right)^{3 / 2} \arctan \sqrt{\frac{b^{2}}{-b_{0}^{2}}}-\frac{s}{b^{2}} \sqrt{\frac{s}{-b_{0}^{2}}}\right), \quad s \simeq 4 m_{\pi}^{2}, \\
& F_{\mu}^{(2)}\left(p_{1},-p_{1}, 0\right)=-\frac{i}{16 \pi^{2}} \frac{p_{1 \mu}}{p_{1}^{2}}\left(-\frac{1}{\lambda^{2}}+\frac{1}{p_{1}^{2}} \ln \left(\frac{m_{\pi}^{2}}{\lambda^{2}}\right)\right) \\
& \bar{R}\left(p_{1}, p_{2}\right)=\frac{i}{\varepsilon}+\frac{2 i}{16 \pi^{2}}\left(1-2 \sqrt{\frac{-b_{0}^{2}}{s}}\left(\frac{\pi}{2}-\arctan \sqrt{\frac{-4 b_{0}^{2}}{s}}\right)\right), \\
& \bar{R}\left(p_{1},-p_{1}\right)=\frac{i}{\varepsilon}
\end{aligned}
$$

One finds for the vertex function the expressions (at leading orders):

$$
\begin{aligned}
\Lambda\left(p_{1}, p_{2}\right)= & \frac{e^{2}}{\varepsilon}(3-(2+\xi))+\frac{\alpha}{2 \pi}+\frac{\alpha}{2} \frac{4 p_{1} \cdot p_{2}}{s} \sqrt{\frac{s}{b^{2}}} \arctan \sqrt{\frac{b^{2}}{-b_{0}^{2}}} \\
& -\frac{\alpha}{2 \pi} \frac{4 p_{1} \cdot p_{2}}{s}\left(2 \ln \left(\frac{s}{\lambda^{2}}\right)-4 \ln 2+4\right)-\frac{\alpha \xi}{2 \pi} \ln \left(\frac{m_{\pi}^{2}}{\lambda^{2}}\right), \quad s \simeq 4 m_{\pi}^{2}, \\
\Lambda\left(p_{1},-p_{1}\right)= & \frac{e^{2}}{\varepsilon}(3-(2+\xi))+\frac{\alpha}{2 \pi}(2+\xi)\left(1-\ln \left(\frac{m_{\pi}^{2}}{\lambda^{2}}\right)-\frac{\lambda^{2}}{p_{1}^{2}} \ln \left(\frac{m_{\pi}^{2}}{\lambda^{2}}\right)\right)
\end{aligned}
$$

Among the integrals involved in the calculation of the constraint diagrams, $F^{(1) C}$ and $\bar{R}^{C}$ were given in Sec. 5 [Eqs. (5.9)-(5.11)]. The integral $F_{\mu}^{(2) C}$ is:

$$
\begin{aligned}
F_{\mu}^{(2) C}\left(p_{1}\right) & =\frac{i}{2 \sqrt{s}} \int \frac{d^{3} k^{T}}{(2 \pi)^{3}} \frac{k_{\mu}^{T}}{\left(\left(p_{1}-k^{T}\right)^{2}-m_{\pi}^{2}+i \epsilon\right)\left(k^{T 2}+i \epsilon\right)^{2}} \\
& =-\frac{1}{4 \pi} \frac{i}{4 \sqrt{s}} p_{\mu}^{T} \frac{1}{\left(b^{2}\right)^{3 / 2}}\left(\arctan \sqrt{\frac{b^{2}}{-b_{0}^{2}}}-\frac{\sqrt{-b^{2} b_{0}^{2}}}{\lambda^{2}}\right) .
\end{aligned}
$$




\section{E The scattering amplitude in the unequal mass case}

In this appendix we present the details of the calculation of the off-mass shell $\pi^{+}\left(p_{1}\right) \pi^{-}\left(p_{2}\right)$ $\rightarrow \pi^{0}\left(p_{3}\right) \pi^{0}\left(p_{4}\right)$ scattering amplitude at $t=u=0$. We need for this evaluation the expression of the function $\bar{R}$ [Eq. (D.10)] in the unequal mass case. Defining:

$$
\bar{R}^{12}(s)=\mu^{4-d} \int \frac{d^{d} k}{(2 \pi)^{d}} \frac{1}{\left(\left(p_{1}-k\right)^{2}-m_{1}^{2}+i \epsilon\right)\left(\left(p_{2}+k\right)^{2}-m_{2}^{2}+i \epsilon\right)},
$$

one has:

$$
\begin{aligned}
& \bar{R}^{12}(s)=\frac{i}{\varepsilon_{1}}+i \bar{J}^{12}(s), \\
& \bar{J}^{12}(s)=\frac{1}{16 \pi^{2}}\left\{2-\frac{s-\left(m_{1}^{2}-m_{2}^{2}\right)}{2 s} \ln \left(\frac{m_{2}^{2}}{m_{1}^{2}}\right)-Q^{12}(s)\right\},
\end{aligned}
$$

with:

$$
Q^{12}(s)=\left\{\begin{array}{l}
+\frac{\sqrt{4 s b_{0}^{2}(s)}}{s}\left[\ln \left(\frac{\sqrt{s-\left(m_{1}-m_{2}\right)^{2}}+\sqrt{s-\left(m_{1}+m_{2}\right)^{2}}}{\sqrt{s-\left(m_{1}-m_{2}\right)^{2}}-\sqrt{s-\left(m_{1}+m_{2}\right)^{2}}}\right)-i \pi\right], \quad\left(m_{1}+m_{2}\right)^{2}<s, \\
+\frac{\sqrt{-4 s b_{0}^{2}(s)}}{s}\left[\pi-\arctan \left(\frac{\sqrt{-4 s b_{0}^{2}(s)}}{s-\left(m_{1}^{2}+m_{2}^{2}\right)}\right), \quad\left(m_{1}-m_{2}\right)^{2}<s<\left(m_{1}+m_{2}\right)^{2},\right. \\
-\frac{\sqrt{4 s b_{0}^{2}(s)}}{s} \ln \left(\frac{\sqrt{\left(m_{1}+m_{2}\right)^{2}-s}+\sqrt{\left(m_{1}-m_{2}\right)^{2}-s}}{\sqrt{\left(m_{1}+m_{2}\right)^{2}-s}-\sqrt{\left(m_{1}-m_{2}\right)^{2}-s}}, \quad s<\left(m_{1}-m_{2}\right)^{2} .\right.
\end{array}\right.
$$

In Eq. (E.2) $\varepsilon_{1}$ is equal to $\varepsilon$ [Eq. (D.6)] with $m_{1}$ replacing $m_{\pi}$..

For small negative values of $s$, denoted now by $t, \bar{R}^{12}$ behaves to first order in $\Delta m^{2} \equiv$ $\left(m_{1}^{2}-m_{2}^{2}\right)$ as:

$$
\bar{R}^{12}(t) \underset{t \rightarrow 0}{=} \frac{i}{\varepsilon_{1}}+\frac{i}{16 \pi^{2}}\left(\Delta m^{2}+\frac{t}{3}\right) \frac{2}{\left(m_{1}+m_{2}\right)^{2}}+O\left(\left(\Delta m^{2}\right)^{2}, t \Delta m^{2}, t^{2}\right) .
$$

The loop contributions with $\pi^{+} \pi^{-}$and $\pi^{0} \pi^{0}$ propagators, respectively, are:

$$
\begin{aligned}
i F_{0}^{4} \mathcal{M}_{\text {loop }}^{+-} & =\frac{1}{2}\left(s-2 \hat{m} B_{0}\right)\left[\left(s+p_{1}^{2}+p_{2}^{2}+2 m_{\pi^{+}}^{2}-8 \hat{m} B_{0}\right) \bar{R}^{+-}(s)+2 T^{+}\right], \\
i F_{0}^{4} \mathcal{M}_{\text {loop }}^{00} & =\frac{1}{2}\left(s-2 \hat{m} B_{0}\right)\left[\left(p_{3}^{2}+p_{4}^{2}+2 m_{\pi^{0}}^{2}-6 \hat{m} B_{0}\right) \bar{R}^{00}(s)+2 T^{0}\right],
\end{aligned}
$$

where $T^{+}$and $T^{0}$ are defined in Eq. (D.1), with the $\pi^{+}$and $\pi^{0}$ masses, respectively.

The sum of the two contributions with a $\pi^{+} \pi^{0}$ loop is, to first order in $\Delta m_{\pi}^{2}$ (regular terms in $t$ and $u$ are neglected):

$$
\begin{aligned}
i F_{0}^{4} \mathcal{M}_{\text {loop }}^{+0}= & \left(p_{1}^{2}+p_{2}^{2}+2 m_{\pi^{+}}^{2}-4 \hat{m} B_{0}\right) T^{+}+\left(p_{3}^{2}+p_{4}^{2}+2 m_{\pi^{0}}^{2}-4 \hat{m} B_{0}\right) T^{0} \\
& +\frac{1}{2}\left[\left(p_{1}^{2}+m_{\pi^{+}}^{2}-2 \hat{m} B_{0}\right)\left(p_{4}^{2}+m_{\pi^{0}}^{2}-2 \hat{m} B_{0}\right)\right.
\end{aligned}
$$




$$
\begin{aligned}
& \left.\quad+\left(p_{2}^{2}+m_{\pi^{+}}^{2}-2 \hat{m} B_{0}\right)\left(p_{3}^{2}+m_{\pi^{0}}^{2}-2 \hat{m} B_{0}\right)\right] \bar{R}^{+0}(t) \\
& +\frac{1}{2}\left[\left(p_{1}^{2}+m_{\pi^{+}}^{2}-2 \hat{m} B_{0}\right)\left(p_{3}^{2}+m_{\pi^{0}}^{2}-2 \hat{m} B_{0}\right)\right. \\
& \left.\quad+\left(p_{2}^{2}+m_{\pi^{+}}^{2}-2 \hat{m} B_{0}\right)\left(p_{4}^{2}+m_{\pi^{0}}^{2}-2 \hat{m} B_{0}\right)\right] \bar{R}^{+0}(u) \\
& +\frac{1}{2}\left(p_{1}^{2}-p_{3}^{2}\right)\left(p_{2}^{2}-p_{4}^{2}\right) \bar{R}^{+0}(t)+\frac{1}{2}\left(p_{1}^{2}-p_{4}^{2}\right)\left(p_{2}^{2}-p_{3}^{2}\right) \bar{R}^{+0}(u) \\
& +\frac{1}{2}\left(p_{1}^{2}+p_{2}^{2}-p_{3}^{2}-p_{4}^{2}\right)\left[\left(\bar{R}^{+0}(t)+\bar{R}^{+0}(u)\right) \Delta m_{\pi}^{2}-\left(T^{+}-T^{0}\right)\right] \\
& -\frac{1}{6}\left(p_{1}^{2}+p_{2}^{2}+p_{3}^{2}+p_{4}^{2}\right)\left[\left(T^{+}+T^{0}\right)+\left(m_{\pi^{+}}^{2}+m_{\pi^{0}}^{2}\right)\left(\bar{R}^{+0}(t)+\bar{R}^{+0}(u)\right.\right. \\
& \left.\left.\quad+\frac{2 i}{16 \pi^{2}}\right)\right]+\frac{1}{6 t}\left(p_{1}^{2}-p_{3}^{2}\right)\left(p_{2}^{2}-p_{4}^{2}\right)\left[\left(T^{+}+T^{0}\right)\right. \\
& \left.\quad-\left(m_{\pi^{+}}^{2}+m_{\pi^{0}}^{2}\right)\left(\bar{R}^{+0}(t)+\frac{i}{16 \pi^{2}}\right)-t\left(\bar{R}^{+0}(t)-\frac{i}{48 \pi^{2}}\right)\right] \\
& +\frac{1}{6 u}\left(p_{1}^{2}-p_{4}^{2}\right)\left(p_{2}^{2}-p_{3}^{2}\right)\left[\left(T^{+}+T^{0}\right)\right. \\
& \left.\quad-\left(m_{\pi^{+}}^{2}+m_{\pi^{0}}^{2}\right)\left(\bar{R}^{+0}(u)+\frac{i}{16 \pi^{2}}\right)-u\left(\bar{R}^{+0}(u)-\frac{i}{48 \pi^{2}}\right)\right] .
\end{aligned}
$$

The tadpole diagrams at the four-pion vertex originate from the following part of the chiral effective lagrangian:

$$
\begin{aligned}
\mathcal{L}_{\text {tad. }}= & \frac{1}{2 F_{0}^{4}}\left(\pi^{02}+2 \pi^{+} \pi^{-}\right)\left(\frac{1}{2} \partial_{\mu} \pi^{02}+\partial_{\mu}\left(\pi^{+} \pi^{-}\right)\right)\left(\frac{1}{2} \partial^{\mu} \pi^{02}+\partial^{\mu}\left(\pi^{+} \pi^{-}\right)\right) \\
& -2 \hat{m} B_{0} \frac{1}{16 F_{0}^{4}}\left(\pi^{02}+2 \pi^{+} \pi^{-}\right)^{3}
\end{aligned}
$$

and yield the contribution:

$$
\begin{aligned}
i F_{0}^{4} \mathcal{M}_{t a d .}=-[ & 3 s T^{0}+4 s T^{+}-\left(s-p_{1}^{2}-p_{2}^{2}-2 m_{\pi^{+}}^{2}\right) T^{+} \\
& \left.-\left(s-p_{3}^{2}-p_{4}^{2}-2 m_{\pi^{0}}^{2}\right) T^{0}\right]+\frac{3}{2} 2 \hat{m} B_{0}\left(3 T^{0}+4 T^{+}\right) .
\end{aligned}
$$

The contribution to the four-point vertex function from the pseudoscalar densities 6.17) and (7.5), concerning its mass shift part, is:

$$
\begin{aligned}
F_{0}^{4} \Delta \mathcal{M}_{P}= & -e^{2} F_{0}^{2}\left(\frac{80}{9}\left(k_{7}+k_{11}\right)+8 k_{8}^{q \gamma}\right)\left(s-2 \hat{m} B_{0}\right)-4 l_{4} \Delta m_{\pi}^{2}\left(s-2 \hat{m} B_{0}\right) \\
& +2 e^{2} F_{0}^{2}\left(k_{7}-2 k_{8}\right)\left(p_{3}^{2}+p_{4}^{2}-2 m_{\pi^{0}}^{2}\right)-4 l_{3} \Delta m_{\pi}^{2}\left(p_{3}^{2}+p_{4}^{2}-m_{\pi^{0}}^{2}\right) .
\end{aligned}
$$

The $O\left(e^{2} p^{2}\right)$ lagrangian (6.16) provides the contribution:

$$
\begin{aligned}
\mathcal{M}^{\left(e^{2} p^{2}\right)}= & \frac{2 e^{2}}{F_{0}^{2}}\left(3 k_{2}-2 k_{4}\right) s-\frac{2 e^{2}}{F_{0}^{2}}\left(2 k_{2}-k_{4}\right)\left(p_{3}^{2}+p_{4}^{2}\right) \\
& -2 e^{2} \frac{2 \hat{m} B_{0}}{F_{0}^{2}}\left(\frac{10}{9}\left(k_{7}+k_{11}\right)-\left(k_{7}-2 k_{8}\right)\right) .
\end{aligned}
$$




\section{References}

[1] S. Weinberg, Phys. Rev. Lett. 17 (1966) 616.

[2] S. Weinberg, Physica A 96 (1979) 327.

[3] J. Gasser and H. Leutwyler, Ann. Phys. (N.Y.) 158 (1984) 142.

[4] J. Gasser and H. Leutwyler, Nucl. Phys. B250 (1985) 465.

[5] J. L. Petersen, CERN Report No 77-04, 1975-1976 (unpublished); Phys. Reports C 2 (1971) 155.

[6] C. D. Froggatt and J. L. Petersen, Nucl. Phys. B129 (1977) 89.

[7] A. Schenk, Nucl. Phys. B363 (1991) 97.

[8] P. Estabrooks and A. D. Martin, Nucl. Phys. B79 (1974) 301.

[9] B. Hyams et al., Nucl. Phys. B64 (1973) 134; W. Hoogland et al., ibid. B69 (1974) 266.

[10] W. Ochs, thesis, Ludwig-Maximilians-Universität, 1973.

[11] L. Rosselet et al., Phys. Rev. D 15 (1977) 574.

[12] M. M. Nagels et al., Nucl. Phys. B147 (1979) 189; O. Dumbrajs et al., Nucl. Phys. B216 (1983) 277.

[13] J. Gasser, The Second DA $\Phi$ NE Physics Handbook, eds. L. Maiani, N. Paver and G. Pancheri (INFN-LNF publication, 1995), p. 215.

[14] D. Morgan and M. R. Pennington, The Second DA $\Phi N E$ Physics Handbook, eds. L. Maiani, N. Paver and G. Pancheri (INFN-LNF publication, 1995), p. 193.

[15] J. Bijnens, G. Colangelo, G. Ecker, J. Gasser and M. Sainio, Phys. Lett. B 374 (1996) 210.

[16] G. Czapek et al., Letter of intent, CERN/SPSLC 92-44.

[17] L. L. Nemenov, Sov. J. Nucl. Phys. 41 (1985) 629. 
[18] L. G. Afanasyev et al., Phys. Lett. B 308 (1993) 200; B 338 (1994) 478.

[19] N. H. Fuchs, H. Sazdjian and J. Stern, Phys. Lett. B 269 (1991) 183.

[20] M. Gell-Mann, R. J. Oakes and B. Renner, Phys. Rev. 175 (1968) 2198; S. Glashow and S. Weinberg, Phys. Rev. Lett. 20 (1968) 224.

[21] J. Stern, H. Sazdjian and N. H. Fuchs, Phys. Rev. D 47 (1993) 3814.

[22] M. Knecht, B. Moussallam and J. Stern, The Second DAФNE Physics Handbook, eds. L. Maiani, N. Paver and G. Pancheri (INFN-LNF publication, 1995), p. 221.

[23] M. Knecht, B. Moussallam, J. Stern and N. H. Fuchs, Nucl. Phys. B 457 (1995) 513; B $471(1996) 445$.

[24] S. Deser, M. L. Goldberger, K. Baumann and W. Thirring, Phys. Rev. 96 (1954) 774.

[25] J. L. Uretsky and T. R. Palfrey, Jr., Phys. Rev. 121 (1961) 1798.

[26] T. L. Trueman, Nucl. Phys. 26 (1961) 57.

[27] S. M. Bilen'kii, Nguyen Van Hieu, L. L. Nemenov and F. G. Tkebuchava, Sov. J. Nucl. Phys. 10 (1969) 469.

[28] G. V. Efimov, M. A. Ivanov and V. E. Lyobovitskii, Sov. J. Nucl. Phys. 44 (1986) 296.

[29] A. A. Bel'kov, V. N. Pervushin and F. G. Tkebuchava, Sov. J. Nucl. Phys. 44 (1986) 300.

[30] U. Moor, G. Rasche and W. S. Woolcock, Nucl. Phys. A 587 (1995) 747; A. Gashi, G. Rasche, G. C. Oades and W. S. Woolcock, preprint IFA-97/13, nucl-th/9704017.

[31] M. Sander, C. Kuhrts and H. V. von Geramb, Phys. Rev. C 53 (1996) 2610.

[32] V. Lyubovitskij and A. Rusetsky, Phys. Lett. B 389 (1996) 181; V. E. Lyubovitskij, E. Z. Lipartia and A. G. Rusetsky, preprint QFT-TSU/97-50, hep-ph/9706244.

[33] E. A. Kuraev, preprint hep-ph/9702327. 
[34] Ph. Droz-Vincent, Lett. Nuovo Cimento 1 (1969) 839; T. Takabayasi, Prog. Theor. Phys. 54 (1975) 563; I. T. Todorov, Dubna Report No. E2-10125, 1976 (unpublished); A. Komar, Phys. Rev. D 18 (1978) 1881; H. Leutwyler and J. Stern, Phys. Lett. 73B (1978) 75; H. W. Crater and P. Van Alstine, Ann. Phys. (N.Y.) 148 (1983) 57; H. Sazdjian, Phys. Rev. D 33 (1986) 3401; A. N. Mitra and I. Santhanam, Few-body Systems 12 (1992) 41; G. Longhi and L. Lusanna, eds., Constraint's Theory and Relativistic Dynamics, proceedings of the Firenze Workshop, 1986 (World Scientific, Singapore, 1987).

[35] A. A. Logunov and A. N. Tavkhelidze, Nuovo Cimento 29 (1963) 380; R. Blankenbecler and R. Sugar, Phys. Rev. 142 (1966) 1051; F. Gross, Phys. Rev. 186 (1969) 1448; M. H. Partovi and E. L. Lomon, Phys. Rev. D 2 (1970) 1999; R. N. Faustov, Theor. Math. Phys. 3 (1970) 478; C. Fronsdal and R. W. Huff, Phys. Rev. D 3 (1971) 933; I. T. Todorov, Phys. Rev. D 3 (1971) 2351; V. B. Mandelzweig and S. J. Wallace, Phys. Lett. B 197 (1987) 469.

[36] G. P. Lepage, Phys. Rev. A 16 (1977) 863.

[37] H. Jallouli and H. Sazdjian, Ann. Phys. (N.Y.) 253 (1997) 376.

[38] E. E. Salpeter and H. A. Bethe, Phys. Rev. 84 (1951) 1232; M. Gell-Mann and F. Low, Phys. Rev. 84 (1951) 350; N. Nakanishi, Suppl. Prog. Theor. Phys. 43 (1969) 1.

[39] R. Barbieri, M. Ciafaloni and P. Menotti, Nuovo Cimento 55A (1968) 701.

[40] S. Love, Ann. Phys. (N.Y.) 113 (1975) 153.

[41] G. T. Bodwin and D. R. Yennie, Phys. Reports 43 (1978) 267; R. Barbieri and E. Remiddi, Nucl. Phys. B141 (1978) 413; W. Buchmüller and E. Remiddi, Nucl. Phys. B162 (1980) 250.

[42] A. Nandy, Phys. Rev. D 5 (1972) 1531.

[43] T. Das, G. S. Guralnik, V. S. Mathur, F. E. Low and J. E. Young, Phys. Rev. Lett. 18 (1967) 759.

[44] G. Ecker, J. Gasser, A. Pich and E. de Rafael, Nucl. Phys. B321 (1989) 311. 
[45] R. Urech, Nucl. Phys. B433 (1995) 234.

[46] L. D. Landau and E. M. Lifshitz, Quantum Mechanics (Pergamon Press, London, 1958), p. 127.

[47] M. A. B. Bég and R. C. Furlong, Phys. Rev. D 31 (1985) 1370.

[48] R. Jackiw, M. A. B. Bég Memorial Volume, eds. A. Ali and P. Hoodbhoy (World Scientific, Singapore, 1991), p. 25.

[49] J. H. Lowenstein, Commun. Math. Phys. 24 (1971) 1; L. S. Brown, Ann. Phys. (N.Y.) 126 (1980) 135.

[50] Ulf-G. Meissner, G. Müller and S. Steininger, preprint KFA-IKP(TH)-1997-06, hepph/9704377 (to appear in Phys. Lett. B).

[51] R. Baur and R. Urech, Phys. Rev. D 53 (1996) 6552; preprint ZU-TH 30/96, hepph/9612328.

[52] J. Bijnens and J. Prades, preprint FTUV/96-69, hep-ph/9610360.

[53] B. Moussallam, preprint IPNO/TH 97-03, hep-ph/9701400. 


\section{Figures}

Fig. 1. A loop diagram and its constraint diagram, denoted by a cross, in the $s$-channel. The sum of the two diagrams is free of singularities in the $s$-channel.

Fig. 2. Constraint diagrams the dominant part of which cancel the ultra-violet divergence of the continuum contribution in second-order perturbation theory. Wavy lines are photons.

Fig. 3. One-photon exchange diagram in the presence of the strong coupling (a) and its constraint diagram (b).

Fig. 4. Two-loop diagrams having constraint diagram counterparts. 


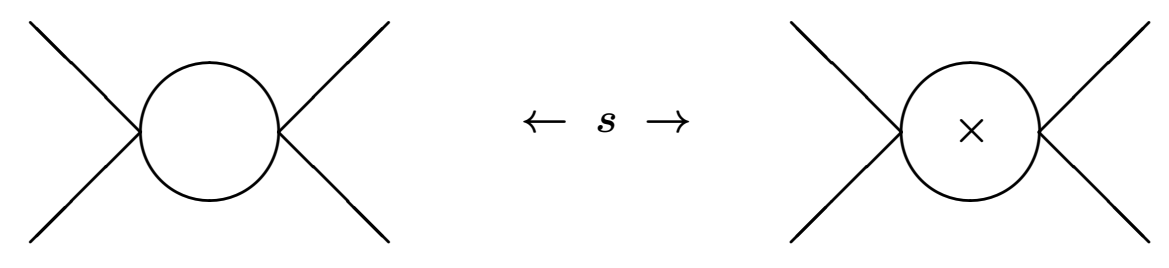

Fig. 1

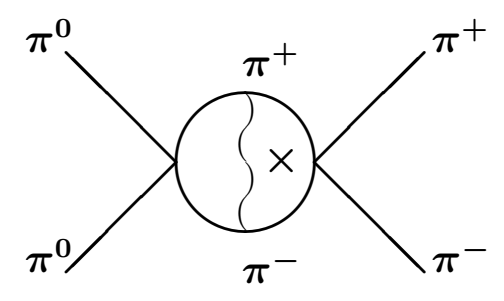

(a)

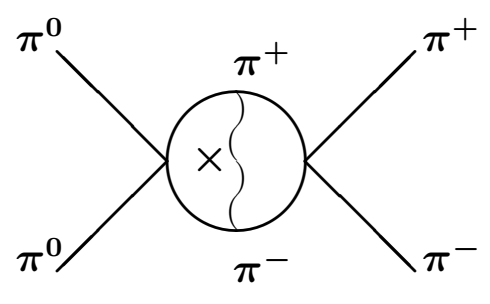

(b)

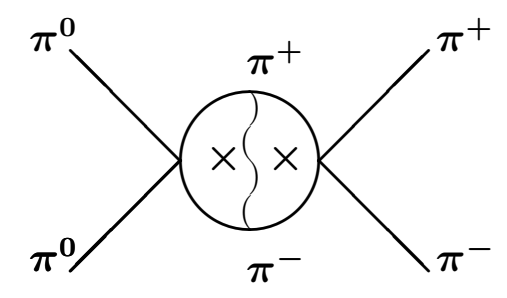

(c)

Fig. 2 


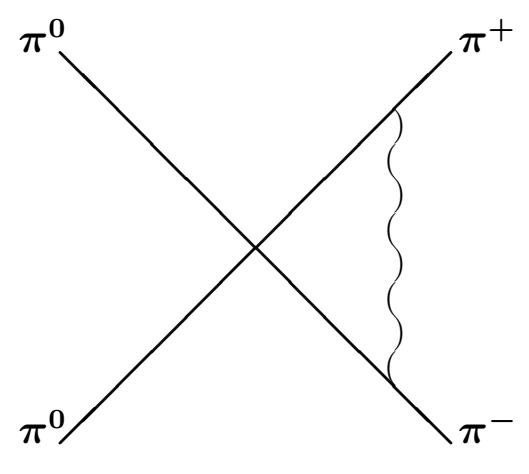

(a)
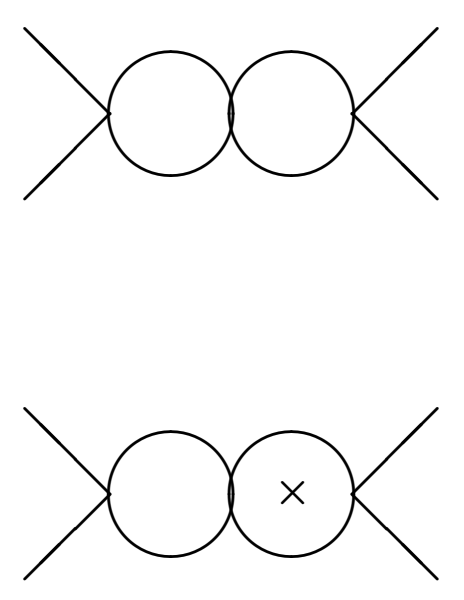

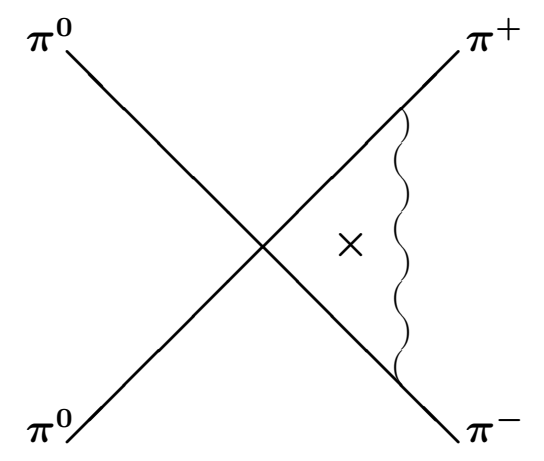

(b)

Fig. 3
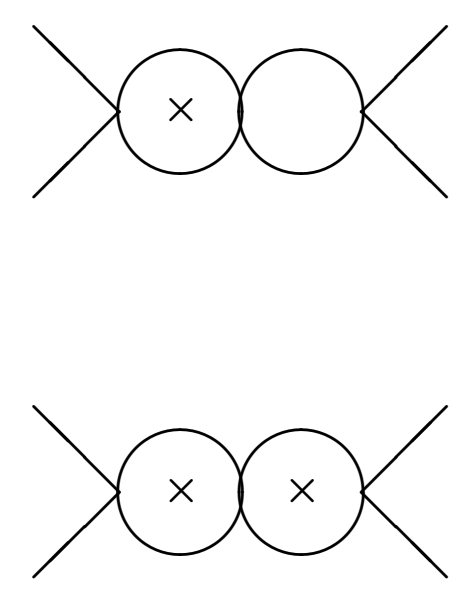

(a) 

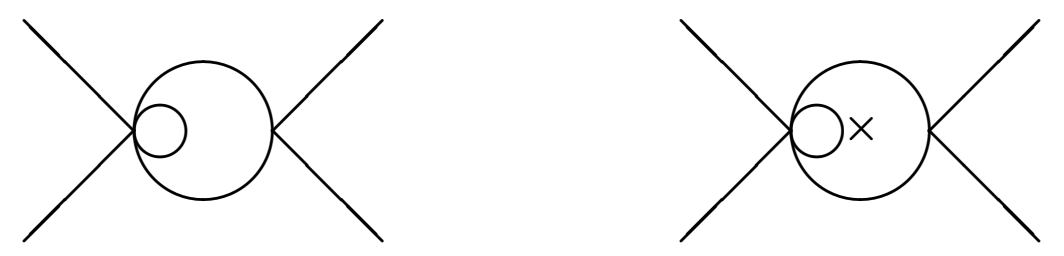

(b)
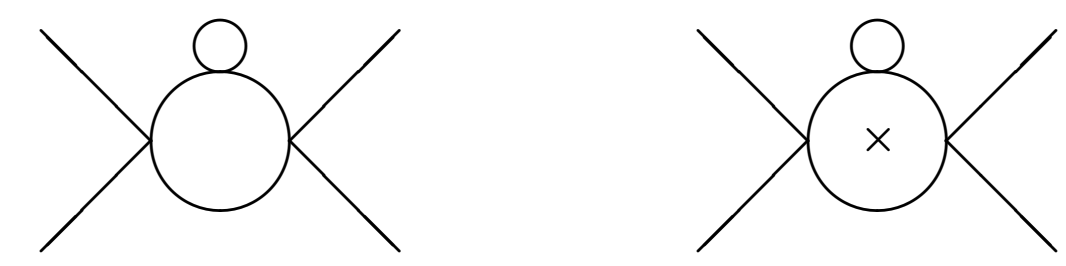

(c)
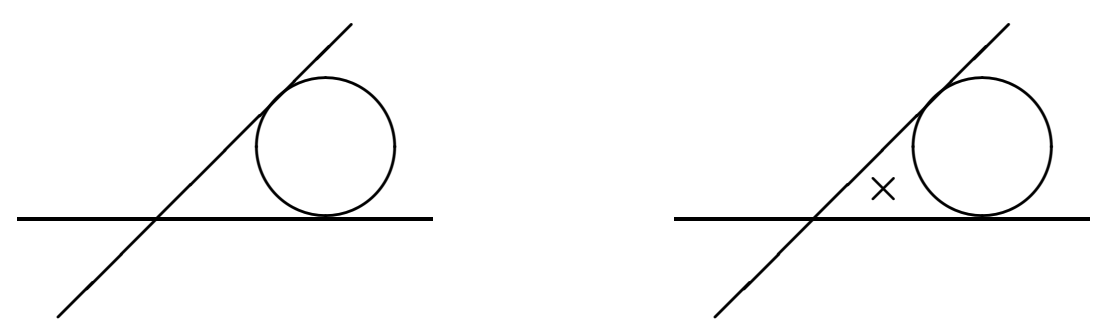

(d)

Fig. 4 